\title{
SYMBOLIC CALCULUS AND FREDHOLM PROPERTY FOR LOCALIZATION OPERATORS
}

\author{
ELENA CORDERO AND KARLHEINZ GRÖCHENIG
}

\begin{abstract}
We study the composition of time-frequency localization operators (wavepacket operators) and develop a symbolic calculus of such operators on modulation spaces. The use of time-frequency methods (phase space methods) allows the use of rough symbols of ultra-rapid growth in place of smooth symbols in the standard classes. As the main application it is shown that, in general, a localization operators possesses the Fredholm property, and thus its range is closed in the target space.
\end{abstract}

\section{INTRODUCTION}

By a symbolic calculus is meant a mapping from a parameter space of symbols to a class of operators and the investigation of this functional dependence. The prototype of a symbolic calculus is the symbolic calculus of pseudodifferential operators. This classical symbolic calculus has lead to an understanding of the composition of pseudodifferential operators, the construction on an approximate inverse, a so-called parametrix, and the regularity properties of partial differential operators $[23,28,30]$.

In this paper we study a different kind of symbolic calculus, namely for (timefrequency) localization operators. This class of operators occurs in various branches of mathematics under such names as Toeplitz operators, (anti) Wick operators, time-frequency multipliers, and others. Their applications range from quantization procedures (Berezin quantization) in quantum mechanics, via signal analysis, to the approximation of pseudodifferential operators. Localization operators form a special case of the wave-packet operators of Cordoba and Fefferman [8] and have been used to approximate general pseudodifferential operators. Technically, localization operators may be regarded as a special class of pseudodifferential operators and may therefore be investigated with pseudodifferential calculus. This approach has led to many interesting results in [7, 26, 31, 32].

Our approach to localization operators is based exclusively on time-frequency methods (phase-space methods). In view of the very definition of this class of operators below, this approach is not only natural, but it also leads to very strong results with respect to the admissible symbols and "window functions". In treating the composition of two localization operators one of the symbols may be rough (in local $L^{\infty}$ ) and even possess ultra-rapid growth.

2000 Mathematics Subject Classification. 35S05,47G30,46E35,47B10.

Key words and phrases. Localization operator, Wick operator, symbolic calculus, modulation space, short-time Fourier transform, Fredholm operator. 
Whereas the product of two pseudodifferential operators is again a pseudodifferential operator (there are many algebras of pseudodifferential operators), the composition of two localization operators is not a localization operator in general. This additional difficulty has captured the interest of several authors and has generated some remarkable ideas. An exact product formula for localization operators is presented in [13]. However, since it works only under very restrictive conditions and is unstable, it is not amenable to a detailed analysis of mapping or compactness properties. Therefore many authors resort to asymptotic expansions that realize the composition of two localization operators as a sum of localization operators and a controllable remainder $[1,11,24,25,29]$. These contributions were mainly motivated by PDEs and energy estimates, and therefore use smooth symbols that are defined by differentiability properties, such as the traditional Hörmander or Shubin classes, and Gaussian windows. For applications in quantum mechanics and signal analysis, alternative notions of smoothness — "smoothness in phasespace" or quantitative measures of "time-frequency concentration" - have turned out to be useful. This point of view is pursued in several recent investigations of localization operators and usually involves modulation spaces (see [11, 31, 32] and references therein). In the context of modulation spaces, much rougher symbols and more general "window functions" can be used for localization operators than have been considered in the studies [10, 12, 27].

Modulation spaces - though still not as well known as standard smoothness spaces - are the appropriate function spaces for time-frequency analysis, and in several cases have been shown to furnish optimal results. For instance, they arise as the optimal symbol classes in the study of boundedness and Schatten class properties of localization operators $[9,10]$

Our analysis of the symbolic calculus for localization operators is based on a composition formula in [1] and (in full generality) in [11]. It was formulated for symbols in certain Shubin classes and windows in the Schwartz class. This calculus seems most suitable for understanding the composition of localization operators with respect to their action on phase-space (the time-frequency plane).

The Short-Time Fourier Transform and Localization Operators. To be more specific and to formulate our results, we first define the short-time Fourier transform and introduce the class of localization operators.

The operators of translation and modulation are defined to be

$$
T_{x} f(t)=f(t-x) \quad \text { and } \quad M_{\omega} f(t)=e^{2 \pi i \omega t} f(t) .
$$

We often combine translations and modulations into time-frequency shifts (phasespace shifts in physical terminology). Write $z=(x, \omega) \in \mathbb{R}^{2 d}$, then the general time-frequency shift is defined by

$$
\pi(z)=M_{\omega} T_{x}
$$

Associated to time-frequency shift is an important transform, the short-time Fourier transform (STFT), which is also called coherent state transform, Gabor transform, windowed Fourier transform, and the like. The STFT of a function or 
distribution $f$ with respect to a fixed non-zero window function $g$ is given by

$$
V_{g} f(x, \omega)=\int_{\mathbb{R}^{d}} f(t) \overline{g(t-x)} e^{-2 \pi i \omega t} d t=\left\langle f, M_{\omega} T_{x} g\right\rangle=\langle f, \pi(z) g\rangle,
$$

whenever the integral or the bracket $\langle\cdot, \cdot\rangle$ is well-defined. The short-time Fourier transform can be defined on many pairs of distribution spaces and test functions. For instance, $V_{g} f$, as a sesquilinear form, maps $L^{2}\left(\mathbb{R}^{d}\right) \times L^{2}\left(\mathbb{R}^{d}\right)$ into $L^{2}\left(\mathbb{R}^{2 d}\right)$ and $\mathcal{S}\left(\mathbb{R}^{d}\right) \times \mathcal{S}\left(\mathbb{R}^{d}\right)$ into $\mathcal{S}\left(\mathbb{R}^{2 d}\right)$. Furthermore, $V_{g} f$ can be extended to a map from $\mathcal{S}^{\prime}\left(\mathbb{R}^{d}\right) \times \mathcal{S}^{\prime}\left(\mathbb{R}^{d}\right)$ into $\mathcal{S}^{\prime}\left(\mathbb{R}^{2 d}\right)$ [18, p. 41]. The short-time Fourier transform is the appropriate tool for defining localization operators.

Next, let $a$ be a symbol on the time-frequency "plane" $\mathbb{R}^{2 d}$ and choose two windows $\varphi_{1}, \varphi_{2}$ on $\mathbb{R}^{d}$, then the localization operator $A_{a}^{\varphi_{1}, \varphi_{2}}$ is defined as

$$
A_{a}^{\varphi_{1}, \varphi_{2}} f(t)=\int_{\mathbb{R}^{2 d}} a(x, \omega) V_{\varphi_{1}} f(x, \omega) M_{\omega} T_{x} \varphi_{2}(t) d x d \omega .
$$

Taking the "inner product" with a test function $g$, the definition of $A_{a}^{\varphi_{1}, \varphi_{2}}$ can be written in a weak sense, namely,

$$
\left\langle A_{a}^{\varphi_{1}, \varphi_{2}} f, g\right\rangle=\left\langle a V_{\varphi_{1}} f, V_{\varphi_{2}} g\right\rangle=\left\langle a, \overline{V_{\varphi_{1}} f} V_{\varphi_{2}} g\right\rangle .
$$

If $a \in \mathcal{S}^{\prime}\left(\mathbb{R}^{2 d}\right)$ and $\varphi_{1}, \varphi_{2} \in \mathcal{S}\left(\mathbb{R}^{d}\right)$, then (5) defines a continuous operator from $\mathcal{S}\left(\mathbb{R}^{d}\right)$ to $\mathcal{S}^{\prime}\left(\mathbb{R}^{d}\right)$. If $\varphi_{1}(t)=\varphi_{2}(t)=2^{d / 4} e^{-\pi t^{2}}$, then $A_{a}=A_{a}^{\varphi_{1}, \varphi_{2}}$ is well-known as (anti-)Wick operator and the mapping $a \rightarrow A_{a}^{\varphi_{1}, \varphi_{2}}$ is interpreted as a quantization rule $[3,13,24,28,33]$. Furthermore, this definition is also a special case of the wave packet operators of Cordoba and Fefferman $[8,16]$, and $A_{a}^{\varphi_{1}, \varphi_{2}}$ serves as an approximation of the pseudodifferential operator $a(x, D)$.

While previous work - to a large extent - uses a combination of methods and is always focused on the model of pseudodifferential operators, we will use exclusively time-frequency analysis in our study of localization operators. In a sense, this is perfectly natural, because the definition of a localization operators is in terms of the basic transform of time-frequency analysis, namely the STFT.

Results. Our starting point is the following composition formula for two localization operators derived in [11]:

$$
A_{a}^{\varphi_{1}, \varphi_{2}} A_{b}^{\varphi_{3}, \varphi_{4}}=\sum_{|\alpha|=0}^{N-1} \frac{(-1)^{|\alpha|}}{\alpha !} A_{a \partial^{\alpha} b}^{\Phi_{\alpha}, \varphi_{2}}+E_{N}
$$

The essence of this formula is that the product of two localization operators can be written as a sum of localization operators and a remainder term, which is "small".

In the spirit of the classical symbolic calculus, this formula was derived in [11, Thm. 1.1] for smooth symbols belonging to some Shubin class $S^{m}\left(\mathbb{R}^{2 d}\right)$ and for windows in the $\mathrm{Schwartz}$ class $\mathcal{S}\left(\mathbb{R}^{d}\right)$. In this case the remainder term is regularizing and maps $L^{2}\left(\mathbb{R}^{d}\right)$ into $\mathcal{S}\left(\mathbb{R}^{d}\right)$.

The goal of this paper is much more ambitious. We will establish the validity of (6) on the modulation spaces (Theorem 6.1 and Proposition 6.2). These function 
spaces are defined by imposing a weighted mixed $L^{p}$-norm on the STFT, e.g., $\|f\|_{M^{p}}=\left\|V_{g} f\right\|_{p}$ (for suitable window $g$ ). In view of definition (5) modulation spaces are bound to arise in the in-depth treatment of the symbolic calculus.

The extension of the composition formula (6) is far from a routine generalization and requires the entire arsenal of time-frequency analysis. The derivation of a symbolic calculus is necessarily technical, and therefore we would like to point out explicitly the innovative features and the level of generality of our results.

(i) Rough symbols. While in (6) the symbol $b$ must be $N$-times differentiable, the symbol $a$ only needs to be locally bounded. The classical results in symbolic calculus require both symbols to be smooth.

(ii) Growth conditions on symbols. The symbolic calculus in (6) can handle symbols with ultra-rapid growth (as long as it is compensated by a decay of $b$ or vice versa). For instance, $a$ may grow subexponentially as $a(z) \sim e^{\alpha|z|^{\beta}}$ for $\alpha>0$ and $0<\beta<1$. This goes far beyond the usual polynomial growth and decay conditions.

(iii) General window classes. We provide a precise description of the admissible windows $\varphi_{j}$ in (6). Usually only the Gaussian $e^{-\pi x^{2}}$ or Schwartz functions are considered as windows $[1,11,24]$.

(iv) Size of the remainder term. We derive norm estimates for the size of the remainder term $E_{N}$ that depend explicitly on the symbols $a, b$ and the windows $\varphi_{j}$. In applications to PDE, e.g. $[23,30]$ it is important that the remainder be regularizing, but this property does not exclude the possibility that $E_{N}$ is large in norm. Therefore norm estimates provide important additional information, see also [24, 25] for more motivation.

The Fredholm Property of Localization Operators. By choosing $N=1$, $\varphi_{1}=\varphi_{2}=\varphi$ with $\|\varphi\|_{2}=1, a(z) \neq 0$, and $b=1 / a$, the composition formula $(6)$ yields the following important special case:

$$
A_{a}^{\varphi, \varphi} A_{1 / a}^{\varphi, \varphi}=A_{1}^{\varphi, \varphi}+R=\mathrm{I}+R
$$

Under mild conditions on $a$ we will show that $R$ is compact and that $A_{a}^{\varphi, \varphi}$ is a Fredholm operator between two modulation spaces $M^{p, q}$ and $M_{m}^{p, q}$ (with different weights), see Theorem 7.1. This theorem is remarkable because it works even for ultra-rapidly growing symbols such as $a(z)=e^{\alpha|z|^{\beta}}$ for $\alpha>0$ and $0<\beta<1$. For comparison, the reduction of localization operators to standard pseudodifferential calculus requires elliptic or hypo-elliptic symbols, and the proof of the Fredholm property works only under severe restrictions, see [7].

Itinerary of the Proof of the Symbolic Calculus. To give the reader some insight how the expansion formula (6) is derived, we sketch the main arguments developed in [11].

The composition of two localization operators can be formally written as

$$
A_{a}^{\varphi_{1}, \varphi_{2}} A_{b}^{\varphi_{3}, \varphi_{4}} f=\int_{\mathbb{R}^{2 d}} \int_{\mathbb{R}^{2 d}} a(y) b(z) V_{\varphi_{3}} f(z)\left\langle\pi(z) \varphi_{4}, \pi(y) \varphi_{1}\right\rangle \pi(y) \varphi_{2} d y d z .
$$


Assuming the symbol $b$ to be $N$-times differentiable, we expand $b(z)$ into a Taylor series around $y$ and obtain

$$
b(z)=\sum_{|\alpha| \leq N} \partial^{\alpha} b(y) \frac{(z-y)^{\alpha}}{\alpha !}+b_{N}(y, z),
$$

where the remainder is given by

$$
b_{N}(y, z)=N \sum_{|\alpha|=N} \int_{0}^{1}(1-t)^{N-1} \partial^{\alpha} b(y+t(z-y)) d t \frac{(z-y)^{\alpha}}{\alpha !} .
$$

For each $\alpha$ with $|\alpha|<N$ we obtain a term of the form

$$
\frac{1}{\alpha !} \int_{\mathbb{R}^{2 d}} a(y) \partial^{\alpha} b(y)\left(\int_{\mathbb{R}^{2 d}}(z-y)^{\alpha} V_{\varphi_{3}} f(z)\left\langle\pi(z) \varphi_{4}, \pi(y) \varphi_{1}\right\rangle d z\right) \pi(y) \varphi_{2} d y .
$$

The inner integral over $z$ is linear in $f$ and "covariant" in $y$, and can be expressed as an $\operatorname{STFT}\left\langle f, \pi(y) \Phi_{\alpha}\right\rangle$, where $\Phi_{\alpha}$ depends on $\varphi_{1}, \varphi_{3}, \varphi_{4}$. Using formulas for the moments of the STFT, this new window was calculated explicitly in [11] to be

$$
\Phi_{\alpha}=\frac{1}{(2 \pi i)^{\left|\alpha_{2}\right|}} \sum_{\beta \leq \alpha}\left(\begin{array}{c}
\alpha \\
\beta
\end{array}\right)(-1)^{\left|\beta_{1}\right|}\left\langle\varphi_{3}, X^{\alpha_{1}-\beta_{1}} \partial^{\alpha_{2}-\beta_{2}} \varphi_{4}\right\rangle X^{\beta_{1}} \partial^{\beta_{2}} \varphi_{1} .
$$

[Note that if $\varphi_{1}, \varphi_{3}, \varphi_{4} \in \mathcal{S}\left(\mathbb{R}^{d}\right)$, then also $\Phi_{\alpha} \in \mathcal{S}\left(\mathbb{R}^{d}\right)$.] Consequently, for the terms $|\alpha|<N$, we obtain the localization operators

$$
\frac{1}{\alpha !} \int_{\mathbb{R}^{2 d}} a(y) \partial^{\alpha} b(y) V_{\Phi_{\alpha}} f(y) \pi(y) \varphi_{2} d y=\frac{1}{\alpha !} A_{a \partial^{\alpha} b}^{\Phi_{\alpha}, \varphi_{2}} f .
$$

The terms corresponding to $|\alpha|=N$ can be collected to a remainder $E_{N}$ given informally by

$$
E_{N} f=\int_{\mathbb{R}^{2 d}} \int_{\mathbb{R}^{2 d}} a(y) b_{N}(y, z) V_{\varphi_{3}} f(z)\left\langle\pi(z) \varphi_{4}, \pi(y) \varphi_{1}\right\rangle \pi(y) \varphi_{2} d y d z .
$$

By summing over $\alpha,|\alpha| \leq N$, we obtain the expansion formula (6). In [11, Thm. 1.1] this formal idea was made rigorous for symbols $a, b$ contained in a Shubin class and windows $\varphi_{j}$ in the Schwartz class.

For the extension of the symbolic calculus (6) to rough symbols and general modulation spaces we need to check that all terms in (6) are well-defined on modulation spaces and then derive norm estimates for their size.

To carry out this plan, we will proceed along the following steps. In Section 2 we introduce the modulation spaces and list some of their main properties. In Section 3 we present further time-frequency tools, such as properties of the STFT. In Section 4 we investigate the mapping properties of localization operators between modulation spaces. These results enable us to give a rigorous meaning to most terms in the symbolic calculus (6). Section 5 is devoted to a careful analysis of the remainder term $E_{N}$, and we will formulate conditions for the boundedness and compactness of $E_{N}$ on modulation spaces. This is the most technical part of the paper. In Section 6 we combine the entire machinery and prove the symbolic calculus (6) in the framework of modulation spaces. In the last Section 7 we 
study the Fredholm property of localization operators. This property is the main application of the symbolic calculus and — at least for us - justifies its many subtle technicalities.

Notation. We define $t^{2}=t \cdot t$, for $t \in \mathbb{R}^{d}$, and $x y=x \cdot y$ is the scalar product on $\mathbb{R}^{d}$. Given a vector $x=\left(x_{1}, \ldots, x_{d}\right) \in \mathbb{R}^{d}$, the partial derivative with respect to $x_{j}$ is denoted by $\partial_{j}=\frac{\partial}{\partial x_{j}}$. Given a multi-index $\alpha=\left(\alpha_{1}, \ldots, \alpha_{d}\right) \geq 0$, i.e., $\alpha \in \mathbb{Z}^{d}$ and $\alpha_{j} \geq 0$, we write $\partial^{\alpha}=\partial_{1}^{\alpha_{1}} \cdots \partial_{d}^{\alpha_{d}}$; moreover, we denote by $X^{\alpha}$ the operator of multiplication: $\left(X^{\alpha} f\right)(t)=\left(t_{1}^{\alpha_{1}} \cdots t_{d}^{\alpha_{d}}\right) f(t)$.

Given a set $K \subseteq \mathbb{R}^{2 d}$, we denote by meas $(K)$ the Lebesgue measure of $K$ and by $\chi_{K}$ the characteristic function of $K$. We use the brackets $\langle f, g\rangle$ to denote the extension to $\mathcal{S}^{\prime}\left(\mathbb{R}^{d}\right) \times \mathcal{S}\left(\mathbb{R}^{d}\right)$ of the inner product $\langle f, g\rangle=\int f(t) \overline{g(t)} d t$ on $L^{2}\left(\mathbb{R}^{d}\right)$. The space of smooth functions with compact support on $\mathbb{R}^{d}$ is denoted by $\mathcal{D}\left(\mathbb{R}^{d}\right)$. We denote by $L^{0}\left(\mathbb{R}^{2 d}\right)$ the space of all $f \in L^{\infty}\left(\mathbb{R}^{2 d}\right)$ that vanish at infinity, i.e., for all $\varepsilon>0$ there exists $R=R(\varepsilon)>0$ such that

$$
|f(z)|<\varepsilon, \quad \text { for a. a. }|z|>R \text {. }
$$

The Fourier transform is normalized to be $\hat{f}(\omega)=\mathcal{F} f(\omega)=\int f(t) e^{-2 \pi i t \omega} d t$.

Throughout the paper, we shall use the notation $A \lesssim B$ to indicate $A \leq c B$ for a suitable constant $c>0$, whereas $A \asymp B$ means that $c^{-1} A \leq B \leq c A$ for some $c \geq 1$. The symbol $B_{1} \hookrightarrow B_{2}$ denotes the continuous embedding of the linear space $B_{1}$ into $B_{2}$.

\section{Modulation Spaces}

2.1. Weight Functions. For the quantitative description of decay properties, we use weight functions on the time-frequency plane. In the sequel $v$ will always be a continuous, positive, even, submultiplicative weight function (in short, a submultiplicative weight), i.e., $v(0)=1, v(z)=v(-z)$, and $v\left(z_{1}+z_{2}\right) \leq v\left(z_{1}\right) v\left(z_{2}\right)$, for all $z, z_{1}, z_{2} \in \mathbb{R}^{2 d}$. We furthermore impose the GRS-condition

$$
\lim _{n \rightarrow \infty} v(n z)^{1 / n}=1, \quad \forall z \in \mathbb{R}^{2 d}
$$

The GRS-condition (introduced by Gelfand-Raikov-Shilov [17]) quantifies the subexponential growth of $v$ in a precise manner. Every weight of the form $v(z)=$ $e^{a|z|^{b}}(1+|z|)^{s} \log ^{r}(e+|z|)$ for parameters $a, r, s \geq 0,0 \leq b<1$ satisfies the GRScondition, whereas the exponential weight $v(z)=e^{a|z|}, a>0$, does not. Finally, we assume that $v$ satisfies the property

$$
\int_{0}^{1} v(t z) d t \lesssim v(z) .
$$

Associated to every submultiplicative weight we consider the class of so-called $v$-moderate weights $\mathcal{M}_{v}$. A positive, even weight function $m$ on $\mathbb{R}^{2 d}$ belongs to $\mathcal{M}_{v}$ if it satisfies the condition

$$
m\left(z_{1}+z_{2}\right) \leq C v\left(z_{1}\right) m\left(z_{2}\right) \quad \forall z_{1}, z_{2} \in \mathbb{R}^{2 d} .
$$


We note that this definition implies that $\frac{1}{v} \lesssim m \lesssim v, m \neq 0$ everywhere, and that $1 / m \in \mathcal{M}_{v}$.

We will often use the polynomial weights $\langle\cdot\rangle^{s}$ defined by

$$
\langle(x, \omega)\rangle^{s}=\langle z\rangle^{s}=\left(1+x^{2}+\omega^{2}\right)^{s / 2}, \quad z=(x, \omega) \in \mathbb{R}^{2 d} \quad s \in \mathbb{R}
$$

and the product weights $\langle\cdot\rangle^{s} \otimes\langle\cdot\rangle^{s}$ signifying $\langle x\rangle^{s}\langle\omega\rangle^{s}$.

\subsection{Modulation Spaces.}

The modulation space norms are a measure of the joint time-frequency distribution of a function or distribution. They occur in many problems in time-frequency analysis and play an increasing role as symbol classes for various types of pseudodifferential operators. For their basic properties we refer, for instance, to [18, Ch. 11-13] and the original literature quoted there.

Let $\varphi_{0}(t)=e^{-\pi t^{2}}$ be the Gaussian and let

$$
\mathcal{S}_{\mathcal{C}}=\operatorname{span}\left\{\pi(z) \varphi_{0}: z \in \mathbb{R}^{2 d}\right\}
$$

be the linear space of all finite linear combination of time-frequency shifts of the Gaussian (the "space of special windows").

Definition 2.1. For any moderate weight $m \in \mathcal{M}_{v}$ and $1 \leq p, q \leq \infty$ we define the modulation space norm on $\mathcal{S}_{\mathcal{C}}$ by

$$
\|f\|_{M_{m}^{p, q}}=\left\|V_{\varphi_{0}} f\right\|_{L_{m}^{p, q}}=\left(\int_{\mathbb{R}^{d}}\left(\int_{\mathbb{R}^{d}}\left|V_{\varphi_{0}} f(x, \omega)\right|^{p} m(x, \omega)^{p} d x\right)^{q / p} d \omega\right)^{1 / q} .
$$

If $p, q<\infty$, the modulation space $M_{m}^{p, q}$ is the norm completion of $\mathcal{S}_{\mathcal{C}}$ in the $M_{m}^{p, q}$ norm. If $p=\infty$ or $q=\infty$, then $M_{m}^{p, q}$ is the completion of $\mathcal{S}_{\mathcal{C}}$ in the $\sigma\left(\mathcal{S}_{\mathcal{C}}, \mathcal{S}_{\mathcal{C}}\right)$ topology. Then by definition, $M_{m}^{p, q}\left(\mathbb{R}^{d}\right)$ is a Banach space. If $p=q$, we write $M_{m}^{p}$ instead of $M_{m}^{p, p}$, and if $m(z) \equiv 1$ on $\mathbb{R}^{2 d}$, then we write $M^{p, q}$ and $M^{p}$ for $M_{m}^{p, q}$ and $M_{m}^{p, p}$.

This definition can be read on several levels of generality. If $v$ and therefore $m \in \mathcal{M}_{v}$ grow polynomially, $m(z)=\mathcal{O}\left(|z|^{N}\right)$ for some $N \geq 0$, then $M_{m}^{p, q}$ consists of all tempered distributions $f \in \mathcal{S}^{\prime}\left(\mathbb{R}^{d}\right)$ such that $V_{\varphi_{0}} f \in L_{m}^{p, q}\left(\mathbb{R}^{2 d}\right)$ with the norm $\|f\|_{M_{m}^{p, q}}=\left\|V_{\varphi_{0}} f\right\|_{L_{m}^{p, q}}$. If $p, q \leq 2$ and $m \geq 1$, then $M_{m}^{p, q}$ is a subspace of $L^{2}\left(\mathbb{R}^{d}\right)$.

However, if $v$ and $m$ grow faster than polynomially, we may need to appeal to the theory of ultra test functions and ultradistributions to get a concrete definition of $M_{m}^{p, q}$ in place of the equivalent definition as an abstract norm completion $[4,15,26]$. For instance, if $v(z)=e^{a|z|^{\gamma}}$ for $\gamma<1$, then $M_{v}^{1} \subseteq \mathcal{S}\left(\mathbb{R}^{d}\right) \subseteq \mathcal{S}^{\prime}\left(\mathbb{R}^{d}\right) \subseteq M_{1 / v}^{\infty}$, and the elements in $M_{m}^{p, q}$ are those "ultra distributions" whose STFT is in $L_{m}^{p, q}$.

We list the some of the main properties of modulation spaces [18, Ch. 11].

Theorem 2.1. Let $1 \leq p, q \leq \infty$, $v$ be a submultiplicative weight as above, and $m \in \mathcal{M}_{v}$.

(i) Then $M_{m}^{p, q}\left(\mathbb{R}^{d}\right)$ is a Banach space, and

$$
\|\pi(z) f\|_{M_{m}^{p, q}} \lesssim v(z)\|f\|_{M_{m}^{p, q}}
$$


(ii) Duality: If $1 \leq p, q<\infty$ and $p^{\prime}=\frac{p}{p-1}$ is the conjugate exponent, then $\left(M_{m}^{p, q}\right)^{*}=M_{1 / m}^{p^{\prime}, q^{\prime}}$.

REMARK: The modulation spaces $M_{m}^{\infty, 1}$ is the dual of $M_{1 / m}^{1,0}$ where $M_{1 / m}^{1,0}$ is the closure of $\mathcal{S}_{\mathcal{C}}$ in the $M_{1 / m}^{1, \infty}$-norm [2]. Similarly, $M_{m}^{1, \infty}=\left(M_{1 / m}^{0,1}\right)^{*}$. Thus duality and weak-* arguments can be used for all indices $p, q$.

The following statement on norm equivalence is crucial and will be used repeatedly. Again $\varphi_{0}(t)=e^{-\pi t^{2}}$.

Theorem 2.2. (i) [18, Lemma 12.1.1] If $f, g \in M_{v}^{1}$, then

$$
\left\|V_{g} f\right\|_{L_{v}^{1}} \lesssim\|f\|_{M_{v}^{1}}\|g\|_{M_{v}^{1}} .
$$

(ii) Equivalent norms [18, Thm. 11.4.2]: If $g \in M_{v}^{1}$ and $f \in M_{m}^{p, q}$, then

$$
\left\|V_{g} f\right\|_{L_{m}^{p, q}} \lesssim\|g\|_{M_{v}^{1}}\|f\|_{M_{m}^{p, q}} \lesssim\left\|V_{g} f\right\|_{L_{m}^{p, q}} .
$$

The estimates in (17) imply that the definition of $M_{m}^{p, q}$ is independent of the window $g$ and that $\left\|V_{g} f\right\|_{L_{m}^{p, q}}$ is an equivalent norm for any $g \in M_{v}^{1}$. To understand the various conditions on the occurring windows, it is helpful to keep in mind that $M_{v}^{1}$ is the maximal class of test functions that works simultaneously for all modulation spaces $M_{m}^{p, q}, 1 \leq p, q \leq \infty$ and all $v$-moderate weights $m$.

Among the modulation spaces occur several well-known function spaces :

(i) $M^{2}\left(\mathbb{R}^{d}\right)=L^{2}\left(\mathbb{R}^{d}\right)$.

(ii) Weighted $L^{2}$-spaces: if $\mu_{s}(x, \omega)=\langle x\rangle^{s}$, then

$$
M_{\mu_{s}}^{2}\left(\mathbb{R}^{d}\right)=L_{s}^{2}\left(\mathbb{R}^{d}\right)=\left\{f: f(x)\langle x\rangle^{s} \in L^{2}\left(\mathbb{R}^{d}\right)\right\} .
$$

(iii) Sobolev spaces: if $\tau_{s}(x, \omega)=\langle\omega\rangle^{s}$, then

$$
M_{\tau_{s}}^{2}\left(\mathbb{R}^{d}\right)=H^{s}\left(\mathbb{R}^{d}\right)=\left\{f: \hat{f}(\omega)\langle\omega\rangle^{s} \in L^{2}\left(\mathbb{R}^{d}\right)\right\} .
$$

(iv) Shubin-Sobolev spaces $[28,6]$ : If $m_{s}(z)=(1+|z|)^{s}=\langle z\rangle^{s}$, then

$$
M_{m_{s}}^{2}\left(\mathbb{R}^{d}\right)=L_{s}^{2}\left(\mathbb{R}^{d}\right) \cap H^{s}\left(\mathbb{R}^{d}\right)=Q_{s}\left(\mathbb{R}^{d}\right)
$$

(v) The Schwartz class is related to modulation spaces as follows $[21]: \mathcal{S}\left(\mathbb{R}^{d}\right)=$ $\bigcap_{s \geq 0} M_{\langle\cdot\rangle}^{\infty}\left(\mathbb{R}^{d}\right)$.

(vi) The space of tempered distributions [21]:

$$
\mathcal{S}^{\prime}\left(\mathbb{R}^{d}\right)=\bigcup_{s \leq 0} M_{\langle\cdot\rangle}^{\infty}\left(\mathbb{R}^{d}\right)
$$

\section{Time-FREQUENCY TOOLS}

The following properties of the STFT, defined in (3), will be used in the sequel. For proofs, see [16], [18, Ch. 3].

Lemma 3.1. Let $f, g, h \in L^{2}\left(\mathbb{R}^{d}\right)$. Then we have

(i) Covariance property: if $x, u, \omega, \eta \in \mathbb{R}^{d}$, then

$$
V_{g}\left(M_{\eta} T_{u} f\right)(x, \omega)=e^{-2 \pi i u(\omega-\eta)} V_{g} f(x-u, \omega-\eta) .
$$

and thus $\left|V_{g}(\pi(y))(z)\right|=\left|V_{g} f(z-y)\right|$ for $y, z \in \mathbb{R}^{2 d}$. 
(ii) Inversion formula:

$$
\int_{\mathbb{R}^{2 d}} V_{g} f(x, \omega) M_{\omega} T_{x} h d x d \omega=\langle h, g\rangle f,
$$

where the integral is to be understood in the weak sense.

Fix a non-zero window $\gamma$, we define an operator $V_{\gamma}^{*}$ mapping a function $F$ on $\mathbb{R}^{2 d}$ to a function or distribution on $\mathbb{R}^{d}$ by

$$
V_{\gamma}^{*} F=\int_{\mathbb{R}^{2 d}} F(x, \omega) M_{\omega} T_{x} \gamma d x d \omega .
$$

If $\gamma, f \in L^{2}\left(\mathbb{R}^{d}\right)$ and $F \in L^{2}\left(\mathbb{R}^{2 d}\right)$, then $V_{\gamma}^{*}$ is simply the adjoint of $V_{\gamma}$ in the sense that

$$
\left\langle V_{\gamma}^{*} F, f\right\rangle=\left\langle F, V_{\gamma} f\right\rangle .
$$

The following proposition [18, Prop. 11.3.2] concerns the boundedness of the operator $V_{\gamma}^{*}$ and will be crucial for the analysis of localization operators.

Proposition 3.1. Let $m \in \mathcal{M}_{v}, \gamma \in M_{v}^{1}, 1 \leq p, q \leq \infty$. Then $V_{\gamma}^{*}$ maps $L_{m}^{p, q}\left(\mathbb{R}^{2 d}\right)$ onto $M_{m}^{p, q}$ and satisfies

$$
\left\|V_{\gamma}^{*} F\right\|_{M_{m}^{p, q}} \lesssim\left\|V_{\varphi_{0}} \gamma\right\|_{L_{v}^{1}}\|F\|_{L_{m}^{p, q}}=\|\gamma\|_{M_{v}^{1}}\|F\|_{L_{m}^{p, q}} .
$$

In particular, if $F=V_{g} f$, then the inversion formula holds in $M_{m}^{p, q}$ :

$$
f=\frac{1}{\langle\gamma, g\rangle} \int_{\mathbb{R}^{2 d}} V_{g} f(x, \omega) M_{\omega} T_{x} \gamma d x d \omega
$$

In this notation, we can write a localization operator informally as

$$
A_{a}^{\varphi_{1}, \varphi_{2}} f=V_{\varphi_{2}}^{*}\left(a V_{\varphi_{1}} f\right) .
$$

Lemma 3.2. Let $v$ be any submultiplicative weight and $\varphi_{0}(t)=e^{-\pi t^{2}}$. Then, for all multi-indices $\alpha, \beta \geq 0$,

$$
\left\|V_{\varphi_{0}}\left(X^{\beta} \partial^{\alpha} \varphi_{0}\right)\right\|_{L_{v}^{1}}=\int_{\mathbb{R}^{2 d}}\left|V_{\varphi_{0}}\left(X^{\beta} \partial^{\alpha} \varphi_{0}\right)(x, \omega)\right| v(x, \omega) d x d \omega<\infty .
$$

Proof. We use the (multivariate) Hermite functions $h_{\gamma}$ to show that the integral (25) is finite. Since $X^{\beta} \partial^{\alpha} \varphi_{0}$ can be written in the form $p \varphi_{0}$ where $p$ is a polynomial of degree $|\alpha|+|\beta|, X^{\beta} \partial^{\alpha} \varphi_{0}$ is a finite sum of Hermite functions $X^{\beta} \partial^{\alpha} \varphi_{0}=\sum_{|\gamma| \leq|\alpha|+|\beta|} c_{\gamma} h_{\gamma}$. By a well-known formula (called the "Laguerre connection" in [16]), the STFT of $h_{\gamma}$ is $V_{\varphi_{0}} h_{\gamma}(x, \omega)=L_{0, \gamma}\left(\pi\left(x^{2}+\omega^{2}\right)\right)(x, \omega) e^{-\pi\left(x^{2}+\omega^{2}\right) / 2}$, where $L_{0, \gamma}$ is a Laguerre polynomial of degree $|\gamma|$. Consequently, $V_{\varphi_{0}}\left(X^{\beta} \partial^{\alpha} \varphi_{0}\right)=$ $|P(x, \omega)| e^{-\pi\left(x^{2}+\omega^{2}\right) / 2}$ for some polynomial of degree at most $|\alpha|+|\beta|$. Since a submultiplicative weight $v$ grows at most exponentially (i.e., $\left.v(x, \omega) \leq \mathcal{O}\left(e^{c(|x|+|\omega|)}\right)\right)$, the convergence of the integral (25) is guaranteed.

Lemma 3.2 helps us prove the boundedness of the operators $X^{\beta} \partial^{\alpha}$ from $M_{v\left(\langle\rangle^{N} \otimes\langle\cdot\rangle^{N}\right)}^{1}$ into $\left.M_{v\left(\langle\rangle^{N-|\beta|}\right.}^{1} \otimes\langle\cdot\rangle^{N-|\alpha|}\right)$. A similar result can be found in [32]. 
Lemma 3.3. Let $g \in M_{v\left(\langle\cdot\rangle^{N} \otimes\langle\cdot\rangle^{N}\right)}^{1}$ and $|\alpha|,|\beta| \leq N$. Then

$$
\left\|X^{\beta} \partial^{\alpha} g\right\|_{\left.M_{v\left(\langle\cdot\rangle^{N-|\beta|}\right.}^{1} \otimes\langle\cdot\rangle^{N-|\alpha|}\right)} \lesssim\|g\|_{M_{v\left(\langle\cdot\rangle^{N} \otimes\langle\cdot\rangle^{N}\right)}^{1}} .
$$

Proof. We use the following algebraic formula [18, Lemma 11.2.1] to interchange the operators $\partial^{\alpha} X^{\beta}$ with time-frequency shifts:

$$
\partial^{\alpha} X^{\beta}\left(M_{\omega} T_{x} h\right)=\sum_{\delta_{1} \leq \alpha} \sum_{\delta_{2} \leq \beta}\left(\begin{array}{c}
\alpha \\
\delta_{1}
\end{array}\right)\left(\begin{array}{c}
\beta \\
\delta_{2}
\end{array}\right) x^{\delta_{2}}(2 \pi i \omega)^{\delta_{1}} M_{\omega} T_{x}\left(\partial^{\alpha-\delta_{1}} X^{\beta-\delta_{2}} h\right),
$$

for all $(x, \omega) \in \mathbb{R}^{2 d}$ and $\alpha, \beta$ with $|\alpha|,|\beta| \leq N$. Substituting (27) into the STFT of $X^{\beta} \partial^{\alpha} g$, we obtain

$$
\begin{aligned}
& \left|V_{\varphi_{0}}\left(X^{\beta} \partial^{\alpha} g\right)(x, \omega)\right|=\left|\left\langle X^{\beta} \partial^{\alpha} g, M_{\omega} T_{x} \varphi_{0}\right\rangle\right|=\left|\left\langle g, \partial^{\alpha} X^{\beta}\left(M_{\omega} T_{x} \varphi_{0}\right)\right\rangle\right| \\
& \leq \sum_{\delta_{1} \leq \alpha} \sum_{\delta_{2} \leq \beta}\left(\begin{array}{l}
\alpha \\
\delta_{1}
\end{array}\right)\left(\begin{array}{c}
\beta \\
\delta_{2}
\end{array}\right)\left|x^{\delta_{2}}(2 \pi i \omega)^{\delta_{1}}\right|\left|\left\langle g, M_{\omega} T_{x}\left(\partial^{\alpha-\delta_{1}} X^{\beta-\delta_{2}} \varphi_{0}\right)\right\rangle\right|
\end{aligned}
$$

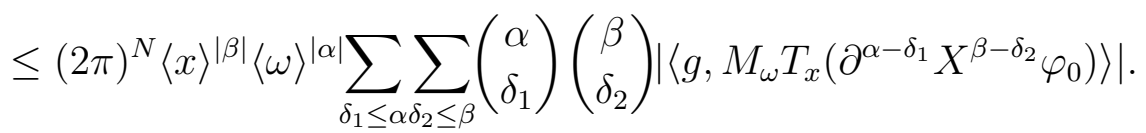

Taking the $L^{1}$-norm with weight $v\left(\langle\cdot\rangle^{N-|\beta|} \otimes\langle\cdot\rangle^{N-|\alpha|}\right)$, the previous estimate yields

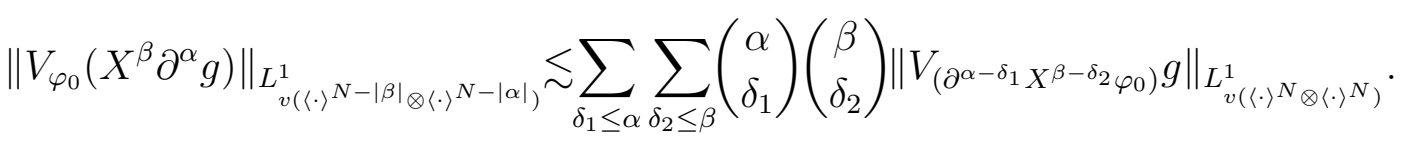

Now we apply Theoerem 2.2(i) to each of the terms on the right-hand side and obtain that

$$
\left\|X^{\beta} \partial^{\alpha} g\right\|_{M_{v\left(\langle\cdot\rangle^{N} \otimes\langle\cdot\rangle^{N}\right)}^{1}} \lesssim \sum_{\delta_{1} \leq \alpha} \sum_{\delta_{2} \leq \beta}\|g\|_{M_{\left.v(\zeta \cdot\rangle^{N} \otimes\langle\zeta\rangle^{N}\right)}^{1}}\left\|\partial^{\alpha-\delta_{1}} X^{\beta-\delta_{2}} \varphi_{0}\right\|_{M_{\left.v(\zeta \cdot\rangle^{N} \otimes\langle\cdot\rangle^{N}\right)}^{1}}<\infty .
$$

The latter expression is finite, since by assumption $g \in M_{v\left(\langle\cdot\rangle^{N} \otimes\langle\cdot\rangle^{N}\right)}^{1}$ and $\partial^{\alpha} X^{\beta} \varphi_{0} \in$ $M_{v\left(\langle\cdot\rangle^{N} \otimes\langle\cdot\rangle^{N}\right)}^{1}$ by Lemma 3.2.

REMARK: Similarly, one can see that $X^{\beta} \partial^{\alpha} \operatorname{maps} M_{m\left(\langle\cdot\rangle^{N} \otimes\langle\cdot\rangle^{N}\right)}^{p, q}$ into $M_{m\left(\langle\cdot\rangle^{N-|\beta|} \otimes\langle\cdot\rangle^{N-|\alpha|}\right)}^{p, q}$

\section{Mapping Properties of Localization Operators Between MOdulation SPACES}

We investigate how a localization operator maps modulation spaces into each others. For a more detailed analysis of the boundedness properties of localization operators we refer to [10].

Theorem 4.1. Let $m \in \mathcal{M}_{v}, \mu \in \mathcal{M}_{w}$.

(i) Assume that $a \in L_{1 / m}^{\infty}\left(\mathbb{R}^{2 d}\right), \varphi_{1} \in M_{v w}^{1}$ and $\varphi_{2} \in M_{w}^{1}$, then the localization operator $A_{a}^{\varphi_{1}, \varphi_{2}}$ is bounded from $M_{\mu m}^{p, q}$ into $M_{\mu}^{p, q}$ with a norm estimate

$$
\left\|A_{a}^{\varphi_{1}, \varphi_{2}} f\right\|_{M_{\mu}^{p, q}} \lesssim\left\|\varphi_{1}\right\|_{M_{v w}^{1}}\left\|\varphi_{2}\right\|_{M_{w}^{1}}\|a\|_{L_{1 / m}^{\infty}}\|f\|_{M_{\mu m}^{p, q}}
$$


(ii) If $a \in L_{m}^{\infty}\left(\mathbb{R}^{2 d}\right), \varphi_{1} \in M_{w}^{1}$, and $\varphi_{2} \in M_{v w}^{1}$, then $A_{a}^{\varphi_{1}, \varphi_{2}}$ is bounded from $M_{\mu}^{p, q}$ into $M_{\mu m}^{p, q}$, with

$$
\left\|A_{a}^{\varphi_{1}, \varphi_{2}} f\right\|_{M_{\mu m}^{p, q}} \lesssim\left\|\varphi_{1}\right\|_{M_{w}^{1}}\left\|\varphi_{2}\right\|_{M_{v w}^{1}}\|a\|_{L_{m}^{\infty}}\|f\|_{M_{\mu}^{p, q} .}
$$

Proof. We write the localization operator $A_{a}^{\varphi_{1}, \varphi_{2}}$ as in (24) and use Proposition 3.1. (i) Since $\varphi_{2} \in M_{w}^{1}$, estimate (22) implies that $V_{\varphi_{2}}^{*}$ is bounded from $L_{\mu}^{p, q}$ onto $M_{\mu}^{p, q}$ and, for $f \in M_{\mu m}^{p, q}$, we have

$$
\begin{aligned}
\left\|A_{a}^{\varphi_{1}, \varphi_{2}} f\right\|_{M_{\mu}^{p, q}} & =\left\|V_{\varphi_{2}}^{*}\left(a V_{\varphi_{1}} f\right)\right\|_{M_{\mu}^{p, q}} \\
& \lesssim\left\|\varphi_{2}\right\|_{M_{w}^{1}}\left\|a V_{\varphi_{1}} f\right\|_{L_{\mu}^{p, q}}
\end{aligned}
$$

Since by assumption $|a(x, \omega)| \leq\|a\|_{L_{1 / m}^{\infty}} m(x, \omega)$ for $(x, \omega) \in \mathbb{R}^{2 d}$, the $L_{\mu}^{p, q}$-norm of the product $a V_{\varphi_{1}} f$ can be controlled in the following way:

$\left\|a V_{\varphi_{1}} f\right\|_{L_{\mu}^{p, q}} \leq\|a\|_{L_{1 / m}^{\infty}}\left\|m V_{\varphi_{1}} f\right\|_{L_{\mu}^{p, q}}=\|a\|_{L_{1 / m}^{\infty}}\left\|V_{\varphi_{1}} f\right\|_{L_{\mu m}^{p, q}} \leq\left\|\varphi_{1}\right\|_{M_{v w}^{1}}\|a\|_{L_{1 / m}^{\infty}}\|f\|_{M_{\mu m}^{p, q}}$

In the last equivalence we have used $\varphi_{1} \in M_{v w}^{1}$ and Theorem $2.2(i i)$.

(ii) is similar. Since $\varphi_{2} \in M_{v w}^{1}, V_{\varphi_{2}}^{*}$ is bounded from $L_{\mu m}^{p, q}$ onto $M_{\mu m}^{p, q}$ by Proposition 3.1. Hence, for $f \in M_{\mu}^{p, q}$, we have

$$
\begin{aligned}
\left\|A_{a}^{\varphi_{1}, \varphi_{2}} f\right\|_{M_{\mu m}^{p, q}} & =\left\|V_{\varphi_{2}}^{*}\left(a V_{\varphi_{1}} f\right)\right\|_{M_{\mu m}^{p, q}} \\
& \lesssim\left\|\varphi_{2}\right\|_{M_{v w}^{1}}\left\|a V_{\varphi_{1}} f\right\|_{L_{\mu m}^{p, q}} \\
& \leq\left\|\varphi_{2}\right\|_{M_{v w}^{1}}\|a\|_{L_{m}^{\infty}}\left\|(1 / m) V_{\varphi_{1}} f\right\|_{L_{\mu m}^{p, q}} \\
& =\left\|\varphi_{2}\right\|_{M_{v w}^{1}}\|a\|_{L_{m}^{\infty}}\left\|V_{\varphi_{1}} f\right\|_{L_{\mu}^{p, q}} \lesssim\left\|\varphi_{1}\right\|_{M_{w}^{1}}\left\|\varphi_{2}\right\|_{M_{w v}^{1}}\|a\|_{L_{m}^{\infty}}\|f\|_{M_{\mu}^{p, q}} .
\end{aligned}
$$

REMARK: Note once again the role of the windows. In (i) the source space $M_{\mu m}^{p, q}$ is measured by the window $\varphi_{1}$, which by Theorem 2.2 has to be in $M_{v w}^{1}$. For the target space $M_{\mu}^{p, q}$ we use $\varphi_{2}$, thus the required condition is $\varphi_{2} \in M_{w}^{1}$.

The following result will be used in the proof of the Fredholm property of $A_{a}^{\varphi_{1}, \varphi_{2}}$ (Corollary 7.2). For weights of polynomial growth, Lemma 4.1 was already observed in [7, Lemma 3.8].

Lemma 4.1. Let $m \in \mathcal{M}_{v}, \mu \in \mathcal{M}_{w}$. If $a \in L^{\infty}\left(\mathbb{R}^{2 d}\right)$ with compact support, and $\varphi_{1} \in M_{v}^{1}, \varphi_{2} \in M_{w}^{1}$, then the localization operator $A_{a}^{\varphi_{1}, \varphi_{2}}$ is compact from $M_{m}^{p, q}$ into $M_{\mu}^{p, q}$, for $1 \leq p, q \leq \infty$.

Proof. We show that $A_{a}^{\varphi_{1}, \varphi_{2}}$ is bounded and compact from $M_{1 / v}^{\infty}$ into $M_{w}^{1}$. The compactness of $A_{a}^{\varphi_{1}, \varphi_{2}}$ from $M_{m}^{p, q}$ into $M_{\mu}^{p, q}$ then follows from the continuous embeddings $M_{m}^{p, q} \hookrightarrow M_{1 / v}^{\infty}$ and $M_{w}^{1} \hookrightarrow M_{\mu}^{p, q}$. We denote the compact support of $a$ by $K \subseteq \mathbb{R}^{2 d}$. 
Let $f \in M_{1 / v}^{\infty}$, then

$$
\begin{aligned}
\left\|A_{a}^{\varphi_{1}, \varphi_{2}} f\right\|_{M_{w}^{1}} & =\left\|V_{\varphi_{2}}^{*}\left(a V_{\varphi_{1}} f\right)\right\|_{M_{w}^{1}} \lesssim\left\|a V_{\varphi_{1}} f\right\|_{L_{w}^{1}} \\
& =\int_{K}|a(z)|\left|V_{\varphi_{1}} f(z)\right| w(z) d z \\
& \lesssim \sup _{z \in K}\left|V_{\varphi_{1}} f(z)\right| \frac{1}{v(z)} \int_{K}|a(z)| v(z) w(z) d z \\
& \lesssim\|f\|_{M_{1 / v}^{\infty}},
\end{aligned}
$$

and so $A_{a}^{\varphi_{1}, \varphi_{2}}$ is bounded.

Let $f_{n} \in M_{1 / v}^{\infty}$ be a bounded sequence that converges weak* to some $f \in M_{1 / v}^{\infty}$. This is equivalent to saying that $V_{\varphi_{1}} f_{n}$ converges uniformly on compact sets of $\mathbb{R}^{2 d}$ to $V_{\varphi_{1}} f$, e.g., by [15, Thm. $\left.4.1 \mathrm{v}\right]$. Consequently,

$$
\left\|A_{a}^{\varphi_{1}, \varphi_{2}}\left(f_{n}-f\right)\right\|_{M_{w}^{1}} \lesssim \sup _{z \in K}\left|V_{\varphi_{1}}\left(f_{n}-f\right)(z)\right| \frac{1}{v(z)} \int_{K}|a(z)| v(z) w(z) d z \rightarrow 0,
$$

and this property implies that $A_{a}^{\varphi_{1}, \varphi_{2}}$ is compact from $M_{1 / v}^{\infty}$ to $M_{w}^{1}$.

For the analysis of the remainder, we will use the well-known fact that every linear operator $A: \mathcal{S} \rightarrow \mathcal{S}^{\prime}$ can be written in the form $A f=V_{\varphi_{2}}^{*}\left(T V_{\varphi_{1}} f\right)$ for a suitable integral operator $T$, see e.g. [16, 20]. The boundedness properties of operators given in this form are derived as in Theorem 4.1.

Lemma 4.2. Let $\varphi_{1}, \varphi_{2} \in M_{w}^{1}\left(\mathbb{R}^{d}\right)$ and $T$ be the integral operator with kernel $K$ on $\mathbb{R}^{4 d}$ (acting on functions $F$ on $\mathbb{R}^{2 d}$ ) defined by $T F(y)=\int_{\mathbb{R}^{2 d}} K(y, z) F(z) d z$ and define the operator $A$ (acting on functions $f$ on $\mathbb{R}^{d}$ ) by

$$
\text { Af }=V_{\varphi_{2}}^{*}\left(T V_{\varphi_{1}} f\right) \text {. }
$$

If $\mu \in \mathcal{M}_{w}$ and $T$ is bounded on $L_{\mu}^{p, q}\left(\mathbb{R}^{2 d}\right), 1 \leq p, q \leq \infty$, then $A$ is bounded on $M_{\mu}^{p, q}\left(\mathbb{R}^{d}\right)$.

Proof. Since $T$ is bounded by assumption and $V_{\varphi_{2}}^{*}$ is bounded by Proposition 3.1, we obtain that

$$
\begin{aligned}
\|A f\|_{M_{\mu}^{p, q}} & =\left\|V_{\varphi_{2}}^{*}\left(T V_{\varphi_{1}} f\right)\right\|_{M_{\mu}^{p, q}} \leq\left\|\varphi_{2}\right\|_{M_{w}^{1}}\left\|T\left(V_{\varphi_{1}} f\right)\right\|_{L_{\mu}^{p, q}} \\
& \leq C_{T}\left\|\varphi_{2}\right\|_{M_{w}^{1}}\left\|V_{\varphi_{1}} f\right\|_{L_{\mu}^{p, q}} \lesssim C_{T}\left\|\varphi_{1}\right\|_{M_{w}^{1}}\left\|\varphi_{2}\right\|_{M_{w}^{1}}\|f\|_{M_{\mu}^{p, q}},
\end{aligned}
$$

where the constant $C_{T}$ is the operator norm of $T$ on $L_{\mu}^{p, q}\left(\mathbb{R}^{2 d}\right)$.

\section{Treatment of Remainder Term}

The mapping properties of localization operators, as studied in the previous section, enable us to understand the left-hand side in the expansion formula (6). We now turn to the investigation of the remainder term $E_{N}$.

An explicit formula for the remainder and its Weyl symbol was derived in [11]. Here we give a different treatment that leads to estimates for the operator norm of $E_{N}$. 
We recall the form of the remainder term from [11] and the introduction (6). Let $b_{N}(y, z)$ be given by

$$
b_{N}(y, z)=N \sum_{|\alpha|=N} \int_{0}^{1}(1-t)^{N-1} \partial^{\alpha} b(y+t(z-y)) d t \frac{(z-y)^{\alpha}}{\alpha !}
$$

then the remainder in (6) is given by the formula

$$
E_{N} f=\int_{\mathbb{R}^{2 d}} \int_{\mathbb{R}^{2 d}} a(y) b_{N}(y, z) V_{\varphi_{3}} f(z)\left\langle\pi(z) \varphi_{4}, \pi(y) \varphi_{1}\right\rangle \pi(y) \varphi_{2} d z d y .
$$

If we introduce the integral operator $T$ with kernel

$$
K(y, z)=a(y) b_{N}(y, z)\left\langle\pi(z) \varphi_{4}, \pi(y) \varphi_{1}\right\rangle,
$$

i.e., $T H(y)=\int_{\mathbb{R}^{2 d}} K(y, z) H(z) d z$, then $E_{N}$ can be written formally as

$$
E_{N} f=V_{\varphi_{2}}^{*}\left(T V_{\varphi_{3}} f\right)
$$

After these preparations we can formulate the following result for the boundedness and compactness of the remainder term.

Theorem 5.1. Let $m \in \mathcal{M}_{v}, \mu \in \mathcal{M}_{w}, 1 \leq p, q \leq \infty$ and assume that $\varphi_{1}, \varphi_{4} \in$ $M_{w v\langle\cdot\rangle^{N}}^{1}\left(\mathbb{R}^{d}\right)$, and $\varphi_{2}, \varphi_{3} \in M_{w}^{1}\left(\mathbb{R}^{d}\right)$.

(i) If $a \in L_{1 / m}^{\infty}\left(\mathbb{R}^{2 d}\right)$ and $\partial^{\alpha} b \in L_{m}^{\infty}\left(\mathbb{R}^{2 d}\right)$ for $|\alpha|=N$, then $E_{N}$ is bounded on $M_{\mu}^{p, q}$ with the following estimate

$\left\|E_{N} f\right\|_{M_{\mu}^{p, q}} \lesssim\|a\|_{L_{1 / m}^{\infty}}\left(\sum_{|\alpha|=N} \frac{1}{\alpha !}\left\|\partial^{\alpha} b\right\|_{L_{m}^{\infty}}\right)\left\|\varphi_{1}\right\|_{M_{v w \zeta \cdot\rangle}^{1}}\left\|\varphi_{2}\right\|_{M_{w}^{1}}\left\|\varphi_{3}\right\|_{M_{w}^{1}}\left\|\varphi_{4}\right\|_{M_{v w \backslash \cdot N}^{1}}\|f\|_{M_{\mu}^{p, q}}$

(ii) If $\partial^{\alpha} b m \in L^{0}\left(\mathbb{R}^{2 d}\right)$, then $E_{N}$ is compact on $M_{\mu}^{p, q}$.

Proof. ( $i$ ) It suffices to show that the integral operator $T$ defined by the kernel $K$ in (32) is bounded on $L_{\mu}^{p, q}\left(\mathbb{R}^{2 d}\right)$. Then $E_{N}$ is bounded on $M_{\mu}^{p, q}$ by Lemma 4.2.

Step 1. An estimate for the kernel $K(y, z)$.

By assumption $|a(y)| \leq\|a\|_{L_{1 / m}^{\infty}} m(y)$ and $\left|\partial^{\alpha} b(y)\right| \leq\left\|\partial^{\alpha} b\right\|_{L_{m}^{\infty}} m(y)^{-1}$. Therefore we find that

$$
\begin{aligned}
\left|a(y) b_{N}(y, z)\right| & =|a(y)| N \sum_{|\alpha|=N}\left|\int_{0}^{1}(1-t)^{N-1} \partial^{\alpha} b(y+t(z-y)) d t \frac{(z-y)^{\alpha}}{\alpha !}\right| \\
& \leq N\|a\|_{L_{1 / m}^{\infty} m} m(y)\langle z-y\rangle^{N} \sum_{|\alpha|=N} \frac{1}{\alpha !}\left\|\partial^{\alpha} b\right\|_{L_{m}^{\infty}} \int_{0}^{1} m(y+t(z-y))^{-1} d t \\
& \leq C N\|a\|_{L_{1 / m}^{\infty} m} m(y)\langle z-y\rangle^{N} \sum_{|\alpha|=N} \frac{1}{\alpha !}\left\|\partial^{\alpha} b\right\|_{L_{m}^{\infty}} \int_{0}^{1} m(y)^{-1} v(t(y-z)) d t \\
& \lesssim\|a\|_{L_{1 / m}^{\infty}} \sum_{|\alpha|=N} \frac{1}{\alpha !}\left\|\partial^{\alpha} b\right\|_{L_{m}^{\infty}} v(y-z)\langle y-z\rangle^{N}
\end{aligned}
$$


where in the last inequality we have used property (12) of the weight $v$. Consequently, if we set $C_{a, b, N}:=\|a\|_{L_{1 / m}^{\infty}} \sum_{|\alpha|=N} \frac{1}{\alpha !}\left\|\partial^{\alpha} b\right\|_{L_{m}^{\infty}}<\infty$, the integral kernel $K$ is dominated by a convolution kernel in the sense that

$$
|K(y, z)| \lesssim C_{a, b, N} v(y-z)\langle y-z\rangle^{N}\left|V_{\varphi_{1}} \varphi_{4}(y-z)\right|
$$

Step 2. Boundedness of the integral operator $T$ on $L_{\mu}^{p, q}\left(\mathbb{R}^{2 d}\right)$. By (35) we obtain that

$$
\begin{aligned}
|T H(y)| & =\left|\int_{\mathbb{R}^{2 d}} K(y, z) H(z) d z\right| \\
& \leq C_{a, b, N} \int_{\mathbb{R}^{2 d}} v(y-z)\langle y-z\rangle^{N}\left|V_{\varphi_{1}} \varphi_{4}(y-z)\right||H(z)| d z \\
& =C_{a, b, N}\left(|H| *\left(v\langle\cdot\rangle^{N}\left|V_{\varphi_{1}} \varphi_{4}\right|\right)\right)(y),
\end{aligned}
$$

If $H \in L_{\mu}^{p, q}\left(\mathbb{R}^{2 d}\right)$, then Young's inequality with weights (e.g. [18, Prop. 11.1.3.]) implies that

$$
\begin{aligned}
\|T H\|_{L_{\mu}^{p, q}} & \leq C_{a, b, N}\left\||H| *\left(v\langle\cdot\rangle^{N}\left|V_{\varphi_{1}} \varphi_{4}\right|\right)\right\|_{L_{\mu}^{p, q}} \\
& \lesssim\|H\|_{L_{\mu}^{p, q}}\left\|\left|V_{\varphi_{1}} \varphi_{4}\right| v\langle\cdot\rangle^{N}\right\|_{L_{w}^{1}} \\
& =\|H\|_{L_{\mu}^{p, q}}\left\|V_{\varphi_{1}} \varphi_{4}\right\|_{L_{v w \zeta \cdot\rangle^{1}}^{1}}
\end{aligned}
$$

Step 3. The boundedness of the remainder $E_{N}$ is now a consequence of Lemma 4.2. Accordingly, we need $\varphi_{2}, \varphi_{3} \in M_{w}^{1}$, whereas by Theorem $2.2\left\|V_{\varphi_{1}} \varphi_{4}\right\|_{L_{v w\langle\cdot\rangle^{N}}} \lesssim$ $\left\|\varphi_{1}\right\|_{M_{v w \nmid\rangle^{N}}^{1}}\left\|\varphi_{4}\right\|_{M_{v w\langle\cdot\rangle^{N}}^{1}}$. By keeping track of all the constants, we find estimate (34).

(ii) Compactness of $E_{N}$.

Step 4. We first show that if $b$ has compact support then $E_{N}$ is a compact operator from $M_{\mu}^{\infty}$ into $M_{\mu}^{1}$. So assume that $\operatorname{supp} b \subseteq K$ for some compact set $K \subseteq \mathbb{R}^{2 d}$. Similar arguments as in item $(i)$ give the boundedness of the integral operator $T$ from $M_{\mu}^{\infty}$ to $M_{\mu}^{1}$. Since by assumption $\partial^{\alpha} b \in L_{m}^{\infty}$, we have

$$
\left|\partial^{\alpha} b(z)\right| \leq\left\|\partial^{\alpha} b\right\|_{L_{m}^{\infty} m}(z)^{-1} \chi_{K}(z)
$$

As in Step 1 above we derive the kernel estimate

$$
\begin{gathered}
|K(y, z)| \lesssim\|a\|_{L_{1 / m}^{\infty}} \sum_{|\alpha|=N} \frac{1}{\alpha !}\left\|\partial^{\alpha} b\right\|_{L_{m}^{\infty}} \int_{0}^{1} v(t(z-y)) \chi_{K}(y+t(z-y)) d t \\
\cdot\langle z-y\rangle^{N}\left|V_{\varphi_{4}} \varphi_{1}(z-y)\right| .
\end{gathered}
$$


Let $H \in L_{\mu}^{\infty}\left(\mathbb{R}^{2 d}\right)$. To estimate $T H$ we apply the preceding computation and also use the $w$-moderateness of $\mu$. This yields the majorization

$$
\begin{aligned}
&\|T H\|_{L_{\mu}^{1}} \leq \int_{\mathbb{R}^{2 d}}\left(\int_{\mathbb{R}^{2 d}}|K(y, z)||H(z)| d z\right) \mu(y) d y \\
& \lesssim \int_{\mathbb{R}^{2 d}} \int_{\mathbb{R}^{2 d}} \int_{0}^{1} v(t(z-y)) \chi_{K}(y+t(z-y)) \mid\langle z-y\rangle^{N} H(z) . \\
& \cdot\left|V_{\varphi_{4}} \varphi_{1}(z-y)\right| \mu(z) w(z-y) d z d y d t .
\end{aligned}
$$

Performing the change of variables $z^{\prime}=z-y, y^{\prime}=y+t(z-y)$ so that $d y d z=d y^{\prime} d z^{\prime}$, and using (12), we get

$$
\begin{aligned}
\|T H\|_{L_{\mu}^{1}} & \lesssim\|H\|_{L_{\mu}^{\infty}} \int_{\mathbb{R}^{2 d}} \int_{\mathbb{R}^{2 d}} \int_{0}^{1} v\left(t z^{\prime}\right) \chi_{K}\left(y^{\prime}\right)\left\langle z^{\prime}\right\rangle^{N}\left|V_{\varphi_{4}} \varphi_{1}\left(z^{\prime}\right)\right| w\left(z^{\prime}\right) d z^{\prime} d y^{\prime} d t \\
& \lesssim\|H\|_{L_{\mu}^{\infty}} \int_{\mathbb{R}^{2 d}} \chi_{K}\left(y^{\prime}\right) d y^{\prime} \int_{\mathbb{R}^{2 d}} v\left(z^{\prime}\right) w\left(z^{\prime}\right)\left\langle z^{\prime}\right\rangle^{N}\left|V_{\varphi_{4}} \varphi_{1}\left(z^{\prime}\right)\right| d z^{\prime} \\
& =\|H\|_{L_{\mu}^{\infty}} \operatorname{meas}(K)\left\|V_{\varphi_{4}} \varphi_{1}\right\|_{L_{v w \zeta \succ}^{1}}
\end{aligned}
$$

To prove the compactness, we need to show that every bounded sequence $f_{n} \in$ $M_{\mu}^{\infty}$ possesses a subsequence $f_{n_{k}}$ such that $E_{N} f_{n_{k}}$ converges in the $M_{\mu}^{1}$-norm. So, assume that $f_{n} \in M_{\mu}^{\infty}=\left(M_{1 / \mu}^{1}\right)^{*}$ and $\left\|f_{n}\right\|_{M_{\mu}^{\infty}} \leq 1$ for all $n \in \mathbb{N}$. By the Theorem of Alaoglu-Bourbaki there is a subsequence $f_{n_{k}}$ that converges in the $w^{*}$-topology to some $f \in M_{\mu}^{\infty}$. After replacing $f_{n_{k}}$ by $f_{n_{k}}-f$, we may assume without loss of generality that $f_{n_{k}} \stackrel{w^{*}}{\rightarrow} 0$. Since $\varphi_{3} \in M_{w}^{1}\left(\mathbb{R}^{d}\right) \subseteq M_{1 / \mu}^{1}\left(\mathbb{R}^{d}\right)$, the $w^{*}$-convergence implies in particular that

$$
\left(V_{\varphi_{3}} f_{n_{k}}\right)(z)=\left\langle f_{n_{k}}, \pi(z) \varphi_{3}\right\rangle \rightarrow 0, \quad \forall z \in \mathbb{R}^{2 d} .
$$

The estimate (36) yields the majorization

$$
\begin{gathered}
\left\|E_{N} f_{n_{k}}\right\|_{M_{\mu}^{1}} \lesssim \int_{\mathbb{R}^{2 d}} \int_{\mathbb{R}^{2 d}} \int_{0}^{1} v(t(z-y)) \chi_{K}(y+t(z-y)) \mid\langle z-y\rangle^{N} V_{\varphi_{3}} f_{n_{k}}(z) \cdot \\
\cdot\left|V_{\varphi_{4}} \varphi_{1}(z-y)\right| \mu(z) w(z-y) d z d y d t .
\end{gathered}
$$

Since $V_{\varphi_{3}} f_{n_{k}}(z) \mu(z) \leq\left\|f_{n_{k}}\right\|_{M_{\mu}^{\infty}} \leq 1$ for all $z \in \mathbb{R}^{2 d}$, the previous integral is bounded (uniformly in $n_{k}$ ) by the function

$$
v(t(z-y)) \chi_{K}(y+t(z-y))\langle z-y\rangle^{N}\left|V_{\varphi_{4}} \varphi_{1}(z-y)\right| w(z-y) .
$$

This function is integrable on $\mathbb{R}^{2 d} \times \mathbb{R}^{2 d} \times[0,1]$ by $(37)$. Thus the hypotheses of Lebesgue's Theorem on dominated convergence are satisfied, and we conclude that $\left\|E_{N} f_{n_{k}}\right\|_{M_{\mu}^{1}} \rightarrow 0$, as desired. So $E_{N}$ is compact from $M_{\mu}^{\infty}$ into $M_{\mu}^{1}$.

Step 5. Since the embeddings $M_{\mu}^{p, q} \hookrightarrow M_{\mu}^{\infty}$ and $M_{\mu}^{1} \hookrightarrow M_{\mu}^{p, q}$ are continuous, it follows that $E_{N}$ is compact from $M_{\mu}^{p, q}$ into $M_{\mu}^{p, q}$.

Step 6. If $\left(\partial^{\alpha} b\right) m \in L^{0}$ for all $|\alpha|=N$, there exist sequences of functions $\rho_{n}^{\alpha}$ with compact support such that

$$
\left\|\left(\partial^{\alpha} b\right) m-\left(\rho_{n}^{\alpha}\right) m\right\|_{L^{\infty}} \rightarrow 0, \quad \forall \alpha,|\alpha|=N .
$$


Let $E_{N}^{n}$ denote the operator obtained by replacing the derivatives $\partial^{\alpha} b$ by their approximations $\rho_{n}^{\alpha}$ in (31). Then by Step $5 E_{N}^{n}: M_{\mu}^{p, q} \rightarrow M_{\mu}^{p, q}$ is a compact operator, and we have

$$
\left\|E_{N}-E_{N}^{n}\right\|_{M_{\mu}^{p, q} \rightarrow M_{\mu}^{p, q}} \lesssim \sum_{|\alpha|=N} \frac{\left\|\partial^{\alpha} b-\rho_{n}^{\alpha}\right\|_{L_{m}^{\infty}}}{\alpha !} \rightarrow 0 .
$$

Being the limit of compact operators, $E_{N}$ is also compact from $M_{\mu}^{p, q}$ into $M_{\mu}^{p, q}$.

\section{The Main Theorem - Symbolic Calculus for Localization OPERATORS}

We now have all the pieces in place to prove the validity of the composition formula for localization operators

$$
A_{a}^{\varphi_{1}, \varphi_{2}} A_{b}^{\varphi_{3}, \varphi_{4}}=\sum_{|\alpha|=0}^{N-1} \frac{(-1)^{|\alpha|}}{\alpha !} A_{a \partial^{\alpha} b}^{\Phi_{\alpha}, \varphi_{2}}+E_{N}
$$

on modulation spaces. According to [11] the windows $\Phi_{\alpha}$ are given explicitly by the formula

$$
\Phi_{\alpha}=\frac{1}{(2 \pi i)^{\left|\alpha_{2}\right|}} \sum_{\beta \leq \alpha}\left(\begin{array}{c}
\alpha \\
\beta
\end{array}\right)(-1)^{\left|\beta_{1}\right|}\left\langle\varphi_{3}, X^{\alpha_{1}-\beta_{1}} \partial^{\alpha_{2}-\beta_{2}} \varphi_{4}\right\rangle X^{\beta_{1}} \partial^{\beta_{2}} \varphi_{1} .
$$

In order to treat (38) on modulation spaces, we need to verify that (a) all occurring windows are in the correct window classes, (b) all the terms are well-defined bounded operators on modulation spaces, and (c) the equality (38) holds true. The proof consists in the careful combination of the main results of Sections 4 and 5 .

The generality of the hypotheses on the admissible weight functions, windows, and symbols makes the conditions somewhat technical and cumbersome. At a first reading one may assume that all weights are of at most polynomial growth. Then it suffices to take all windows $\varphi_{j}, j=1, \ldots, 4$, in $\mathcal{S}\left(\mathbb{R}^{d}\right.$ ) (whereas the more general conditions in the following theorem permit to choose them in much larger modulation spaces).

Theorem 6.1. Consider the following set of hypotheses:

(i) Weights: $m \in \mathcal{M}_{v}, \mu \in \mathcal{M}_{w}$.

(ii) Windows: $\varphi_{1}, \varphi_{4} \in M_{v w\left(\langle\cdot\rangle^{N} \otimes\langle\cdot\rangle^{N}\right)}^{1}, \varphi_{2}, \varphi_{3} \in M_{w}^{1}$.

(iii) Symbols: $a \in L_{1 / m}^{\infty}\left(\mathbb{R}^{2 d}\right), \partial^{\alpha} b \in L_{m}^{\infty}\left(\mathbb{R}^{2 d}\right)$ for all $|\alpha| \leq N$.

If hypotheses (i) - (iii) hold, then the symbolic calculus for localization operators is valid in the following sense:

(i) For $|\alpha|<N$ the window $\Phi_{\alpha}$ on $\mathbb{R}^{d}$ defined by (39) belongs to $M_{v w}^{1}$.

(ii) The composition formula given in (38) is well-defined on every $M_{\mu}^{p, q}$, for $1 \leq$ $p, q \leq \infty$. This means that the product on the left-hand side and all operators on the right-hand side are bounded from $M_{\mu}^{p, q}$ to $M_{\mu}^{p, q}$, and (38) is valid as an identity of operators. 
(iii) If, in addition,

$$
\left(\partial^{\alpha} b\right) m \in L^{0}\left(\mathbb{R}^{2 d}\right), \quad \forall \alpha,|\alpha|=N,
$$

then the remainder $E_{N}$ is compact on $M_{\mu}^{p, q}$.

Proof. Boundedness of the operators on the left-hand side. We apply Theorem 4.1 whose assumptions are tailored for this purpose. By (29) $A_{b}^{\varphi_{3}, \varphi_{4}}$ is bounded from $M_{\mu}^{p, q}$ into $M_{\mu m}^{p, q}$, and by (28) $A_{a}^{\varphi_{1}, \varphi_{2}}$ is bounded from $M_{\mu m}^{p, q}$ into $M_{\mu}^{p, q}$. Thus product $A_{a}^{\varphi_{1}, \varphi_{2}} A_{b}^{\varphi_{3}, \varphi_{4}}$ is therefore bounded on $M_{\mu}^{p, q}$ with an operator norm not exceeding $\|a\|_{L_{1 / m}^{\infty}}\|b\|_{L_{m}^{\infty}}\left\|\varphi_{1}\right\|_{M_{v w}^{1}}\left\|\varphi_{2}\right\|_{M_{w}^{1}}\left\|\varphi_{3}\right\|_{M_{w}^{1}}\left\|\varphi_{4}\right\|_{M_{v w}^{1}}$.

Boundedness of the operators on the right-hand side. The boundedness of the remainder $E_{N}$ on $M_{\mu}^{p, q}$ was stated and proved in Theorem 5.1(i). With the additional decay condition (40) the compactness of $E_{N}$ follows from Theorem 5.1(ii). Note that $\langle(x, \omega)\rangle^{N} \leq\langle x\rangle^{N}\langle\omega\rangle^{N}$ and thus $M_{v w\left(\langle\cdot\rangle^{N} \otimes\langle\cdot\rangle^{N}\right)}^{1} \subseteq M_{v w\langle\cdot\rangle^{N}}^{1}$. The slightly stronger condition on $\varphi_{1}, \varphi_{4}$ is required in the next step.

Next we turn to the sum of localization operators $A_{a \partial^{\alpha} b}^{\Phi_{\alpha}, \varphi_{2}}$. We first check that each window $\Phi_{\alpha}$ defined in (39) belongs to $M_{v w}^{1}$. For $\beta=\left(\beta_{1}, \beta_{2}\right)$ and $|\beta| \leq N$, Lemma 3.3 gives

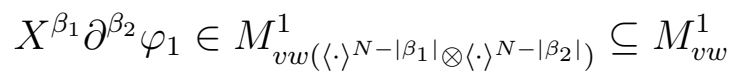

and likewise $X^{\alpha_{1}-\beta_{1}} \partial^{\alpha_{2}-\beta_{2}} \varphi_{4} \in M_{v w}^{1} \subseteq L^{2}$ when $|\beta| \leq|\alpha| \leq N$. Consequently the brackets $\left\langle\varphi_{3}, t^{\alpha_{1}-\beta_{1}} \partial^{\alpha_{2}-\beta_{2}} \varphi_{4}\right\rangle$ are well-defined as an inner product in $L^{2}$, and $\Phi_{\alpha}$, as a finite linear combination of $X^{\beta_{1}} \partial^{\beta_{2}} \varphi_{1}$, is then also in $M_{v w}^{1} \subseteq M_{w}^{1}$.

For boundedness of $A_{a \partial^{\alpha} b}^{\Phi_{\alpha}, \varphi_{2}}$ on $M_{\mu}^{p, q}$ we invoke once more Theorem 4.1. We know that $a \partial^{\alpha} b \in L_{1 / m}^{\infty} \cdot L_{m}^{\infty}=L^{\infty}\left(\mathbb{R}^{2 d}\right)$ and $\Phi_{\alpha}, \varphi_{2} \in M_{w}^{1}$, hence $A_{a \partial^{\alpha} b}^{\Phi_{\alpha}, \varphi_{2}}$ is bounded on $M_{\mu}^{p, q}$.

Formula (38) is an identity of operators. So far we have shown that all terms in the symbolic calculus (38) are well-defined and bounded on $M_{\mu}^{p, q}$. It remains to show that (38) is actually an identity of operators, under the general hypothesis stated. Regarding this question, we already know from the main result in [11] that (38) holds for windows $\varphi_{j}$ in the Schwartz class and symbols in certain Shubin classes, in particular for symbols $a, b \in \mathcal{S}\left(\mathbb{R}^{2 d}\right)$. We need to extend the validity of (38) to windows in the (possibly larger) modulation spaces and to non-smooth symbols in weighted $L^{\infty}$-spaces.

To accomplish this extension, we view each of the terms in the symbolic calculus as a multilinear form mapping $\left(a, b, \varphi_{1}, \ldots, \varphi_{4}\right)=(a, b, \vec{\varphi})$ to one of the operators

$$
T(a, b, \vec{\varphi})=A_{a}^{\varphi_{1}, \varphi_{2}} A_{b}^{\varphi_{3}, \varphi_{4}}, A_{a \partial^{\alpha} b}^{\Phi_{\alpha}, \varphi_{2}}, \text { or } E_{N}(a, b, \varphi) .
$$
form

The operator norm of each of these operators on $M_{\mu}^{p, q}$ obeys an estimate of the

$$
\begin{aligned}
& \|T(a, b, \vec{\varphi})\|_{M_{\mu}^{p, q} \rightarrow M_{\mu}^{p, q}} \lesssim \\
& \quad \lesssim\|a\|_{L_{1 / m}^{\infty}}^{\infty}\left\|\partial^{\alpha} b\right\|_{L_{m}^{\infty}}^{\infty}\left\|\varphi_{1}\right\|_{M_{\left.v w(\zeta \cdot\rangle^{N} \otimes\langle\cdot\rangle^{N}\right)}^{1}}\left\|\varphi_{2}\right\|_{M_{w}^{1}}\left\|\varphi_{3}\right\|_{M_{w}^{1}}\left\|\varphi_{4}\right\|_{M_{\left.\left.v w(\zeta \cdot)^{N} \otimes \zeta \cdot\right\rangle^{N}\right)}^{1}}
\end{aligned}
$$


for suitable $\alpha,|\alpha| \leq N$ as proved in Theorem 4.1 and 5.1, and (34).

The extension of the symbolic calculus from windows in $\mathcal{S}\left(\mathbb{R}^{d}\right)$ to windows in the modulation spaces can be done by a routine density argument, because $\mathcal{S}_{\mathcal{C}}$ is dense in $M_{v}^{1}$ for any submultiplicative weight $v$. Since by $(42)$ each operator $T(a, b, \vec{\varphi})$ is jointly continuous in $\varphi_{j}, j=1, \ldots, 4$, we may choose four sequences $\varphi_{j, n} \in \mathcal{S}_{\mathcal{C}}$ such that $\varphi_{j, n} \rightarrow \varphi_{j}$ in the correctly weighted $M^{1}$-norm. Then, as $n \rightarrow \infty$, each $T\left(a, b, \overrightarrow{\varphi_{n}}\right)$ converges to $T(a, b, \vec{\varphi})$ in operator norm. As a consequence, the symbolic calculus (38) holds under the general hypotheses on the windows as stated.

The extension of the symbolic calculus to non-smooth symbols is more subtle, because $\mathcal{S}$ is not norm-dense in $L_{1 / m}^{\infty}$ or $L_{m}^{\infty}$. We have to take recourse to a weak-* approximation argument.

Given $a \in L_{1 / m}^{\infty}\left(\mathbb{R}^{2 d}\right)$, we choose a sequence $a_{k} \in \mathcal{S}\left(\mathbb{R}^{2 d}\right)$ converging weak- ${ }^{*}$ to $a$, i.e., $\left\langle a_{k}, F\right\rangle \rightarrow\langle a, F\rangle$ for all $F \in L_{m}^{1}$. Likewise, given $b \in L_{m}^{\infty}$ with $\partial^{\alpha} b \in L_{m}^{\infty}$ for all $\alpha,|\alpha| \leq N$, we may choose a sequence $b_{n} \in \mathcal{S}$, such that $\left\langle\partial^{\alpha} b_{n}, F\right\rangle \rightarrow\left\langle\partial^{\alpha} b, F\right\rangle$ for all $F \in L_{1 / m}^{1}$. This is always possible by a regularization of $b$, see e.g. [22].

Next we show that, for each operator $T(a, b, \vec{\varphi})$ in (41) and all $f \in M_{\mu}^{p, q}$ and $g \in M_{1 / \mu}^{p^{\prime}, q^{\prime}}$, we have

$$
\lim _{k, n \rightarrow \infty}\left\langle T\left(a_{k}, b_{n}, \vec{\varphi}\right) f, g\right\rangle=\langle T(a, b, \vec{\varphi}) f, g\rangle .
$$

To be specific, we carry out the argument for the remainder $E_{N}(a, b, \vec{\varphi})$.

Since by Theorem 5.1 we have

$$
\left|\left\langle E_{N}(a, b) f, g\right\rangle\right| \lesssim\|a\|_{L_{1 / m}^{\infty}},
$$

there exists an $F \in L_{m}^{1}$ (depending on all other parameters $b, \varphi_{j}, f, g$ ), such that

$$
\left\langle E_{N}(a, b) f, g\right\rangle=\langle a, F\rangle .
$$

In fact, according to (31) $F$ is given explicitly by

$$
F(y)=\int_{\mathbb{R}^{2 d}} b_{N}(y, z) V_{\varphi_{3}} f(z)\left\langle\pi(z) \varphi_{4}, \pi(y) \varphi_{1}\right\rangle \overline{V_{\varphi_{2}} g(y)} d z
$$

and can be shown directly to be in $L_{m}^{1}\left(\mathbb{R}^{2 d}\right)$ as in Steps 1 and 2 of the proof of Theorem 5.1.

Consequently, if $a_{k} \stackrel{w^{*}}{\rightarrow} a$, then

$$
\left\langle E_{N}\left(a_{k}, b\right) f, g\right\rangle=\left\langle a_{k}, F\right\rangle \rightarrow\langle a, F\rangle=\left\langle E_{N}(a, b) f, g\right\rangle .
$$

For the convergence in $b$, let $E_{N}^{\alpha}$ be the term in the remainder that corresponds to the $\alpha$-th derivative of $b$. Since as part of the proof of Theorem 5.1 we have shown that

$$
\left|\left\langle E_{N}^{\alpha}(a, b) f, g\right\rangle\right| \lesssim\left\|\partial^{\alpha} b\right\|_{L_{m}^{\infty}},
$$

there exists $G \in L_{1 / m}^{1}$ (depending on $a, f, g, \varphi_{j}$ ) such that

$$
\left\langle E_{N}^{\alpha}(a, b) f, g\right\rangle=\left\langle\partial^{\alpha} b, G\right\rangle .
$$

Consequently, if $\partial^{\alpha} b_{n} \stackrel{w^{*}}{\rightarrow} \partial^{\alpha} b$, then

$$
\left\langle E_{N}^{\alpha}\left(a, b_{n}\right) f, g\right\rangle=\left\langle\partial^{\alpha} b_{n}, G\right\rangle \rightarrow\left\langle\partial^{\alpha} b, G\right\rangle=\left\langle E_{N}^{\alpha}(a, b) f, g\right\rangle .
$$


By summing over all $\alpha,|\alpha|=N$, we have shown (43) for $E_{N}$. The weak-* convergence of $A_{a}^{\varphi_{1}, \varphi_{2}} A_{b}^{\varphi_{3}, \varphi_{4}}$ and $A_{a \partial^{\alpha} b}^{\Phi_{\alpha}, \varphi_{2}}$ is shown by exactly the same argument.

Finally, the weak-* convergence of each term in the symbolic calculus (38) implies that it remains valid under weak-* limits of the symbols $a$ and $b$. Consequently, (38) holds for arbitrary symbols $a \in L_{1 / m}^{\infty}$ and $b$ with $\partial^{\alpha} b \in L_{m}^{\infty}$ for $|\alpha| \leq N$. This completes the proof of the expansion formula on $M_{\mu}^{p, q}$.

Theorem 6.1 possesses a symmetric version that is obtained by using the Taylor expansion for the symbol $a$ instead of $b$. Precisely, the following result holds.

Proposition 6.2. Under the assumptions of Theorem 6.1, with the rules of the symbols $a$ and $b$ interchanged, that is, $b \in L_{1 / m}^{\infty}, \partial^{\alpha} a \in L_{m}^{\infty}, \forall \alpha \leq N$, we obtain the symmetric composition formula

$$
A_{a}^{\varphi_{1}, \varphi_{2}} A_{b}^{\varphi_{3}, \varphi_{4}}=\sum_{|\alpha|=0}^{N-1} \frac{(-1)^{|\alpha|}}{\alpha !} A_{\left(\partial^{\alpha} a\right) b}^{\varphi_{3}, \Psi_{\alpha}}+\widetilde{E}_{N} .
$$

Here

$$
\Psi_{\alpha}=\frac{(-1)^{\alpha}}{(2 \pi i)^{\left|\alpha_{2}\right|}} \sum_{\beta \leq \alpha}\left(\begin{array}{c}
\alpha \\
\beta
\end{array}\right)(-1)^{\left|\beta_{1}\right|}\left\langle\varphi_{2}, X^{\alpha_{1}-\beta_{1}} \partial^{\alpha_{2}-\beta_{2}} \varphi_{1}\right\rangle X^{\beta_{1}} \partial^{\beta_{2}} \varphi_{4} .
$$

and the remainder $\widetilde{E}_{N}$ is given by

$$
\widetilde{E}_{N} f=\int_{\mathbb{R}^{2 d}} \int_{\mathbb{R}^{2 d}} a_{N}(z, y) b(z) V_{\varphi_{3}} f(z)\left\langle\pi(z) \varphi_{4}, \pi(y) \varphi_{1}\right\rangle \pi(y) \varphi_{2} d y d z,
$$

with $a_{N}$ already defined in (30). Formula (44) is a well-defined identity of bounded operators on $M_{\mu}^{p, q}$.

(ii) If

$$
\left(\partial^{\alpha} a\right) m \in L^{0}\left(\mathbb{R}^{2 d}\right), \quad \forall \alpha,|\alpha|=N
$$

then $\widetilde{E}_{N}$ is compact on $M_{\mu}^{p, q}$.

\section{Fredholm Property of Localization Operators}

In the final section we investigate the Fredholm property of localization operators. As in the theory of partial differential equations, the construction of a parametrix (a left inverse or a right inverse modulo regularizing terms) is one of the main applications of the symbolic calculus.

Recall that a bounded operator $A: B_{1} \rightarrow B_{2}$ between two Banach spaces $B_{1}, B_{2}$ is called a Fredholm operator if

$$
\operatorname{dim} \operatorname{Ker} A<\infty \quad \text { and } \operatorname{dim} \text { Coker } A<\infty .
$$

Equivalently, $A$ is Fredholm, if there exists a left parametrix $B: B_{2} \rightarrow B_{1}$ such that $B A=\mathrm{I}_{B_{1}}+K_{1}$ for some compact operator $K_{1}: B_{1} \rightarrow B_{1}$ and a right parametrix $C: B_{2} \rightarrow B_{1}$ such that $A C=\mathrm{I}_{B_{2}}+K_{2}$ for a compact operator $K_{2}: B_{2} \rightarrow B_{2}$ $[5,11]$. 
The expansion formula (6) for $A_{a}^{\varphi_{1}, \varphi_{2}}$ with $N=1$ and $b=1 / a$ yields a natural candidate for a parametrix, namely the localization operator $A_{1 / a}^{\varphi_{2}, \varphi_{1}}$. This idea is formulated precisely in the next theorem.

Theorem 7.1 (Fredholm property). Let $m \in \mathcal{M}_{v}, \mu \in \mathcal{M}_{w}$. Assume that the symbol $a$ and the windows $\varphi_{1}, \varphi_{2}$ satisfy the following conditions:

(i) $|a| \asymp 1 / m$, in particular $a \in L_{m}^{\infty}\left(\mathbb{R}^{2 d}\right)$,

(ii) $\left(\partial_{j} a\right) m \in L^{0}$ for $j=1, \ldots, 2 d$, and

(iii) $\varphi_{1}, \varphi_{2} \in M_{w v^{2}(\langle\cdot\rangle \otimes\langle\cdot\rangle)}^{1}$ and $\left|\left\langle\varphi_{1}, \varphi_{2}\right\rangle\right|=1$.

Then the operators

$$
A_{a}^{\varphi_{1}, \varphi_{2}}: M_{\mu}^{p, q} \rightarrow M_{\mu m}^{p, q} \quad \text { and } \quad A_{1 / a}^{\varphi_{2}, \varphi_{1}}: M_{\mu m}^{p, q} \rightarrow M_{\mu}^{p, q}
$$

are Fredholm operators.

Proof. Construction of a left parametrix for $A_{a}^{\varphi_{1}, \varphi_{2}}$. Once more, Theorem 4.1(ii) implies that $A_{a}^{\varphi_{1}, \varphi_{2}}$ is bounded from $M_{\mu}^{p, q}$ into $M_{\mu m}^{p, q}$, since $a \in L_{m}^{\infty}\left(\mathbb{R}^{2 d}\right)$. Likewise, $1 / a \in L_{1 / m}^{\infty}$ and by Theorem $4.1(i) A_{1 / a}^{\varphi_{2}, \varphi_{1}}$ is bounded from $M_{\mu m}^{p, q}$ into $M_{\mu}^{p, q}$.

Next, we apply Theorem 6.1 with $N=1$ to the product $A_{1 / a}^{\varphi_{2}, \varphi_{1}} A_{a}^{\varphi_{1}, \varphi_{2}}$ and obtain the composition formula

$$
A_{1 / a}^{\varphi_{2}, \varphi_{1}} A_{a}^{\varphi_{1}, \varphi_{2}}=A_{1}^{\Phi_{0}, \varphi_{1}}+E_{1}
$$

as an operator identity on $M_{\mu}^{p, q}$.

According to (39), $\Phi_{0}$ is given by $\Phi_{0}=\left\langle\varphi_{1}, \varphi_{2}\right\rangle \varphi_{2}$. Using the inversion formula (20), which corresponds to $a \equiv 1$, we obtain

$$
\begin{aligned}
A_{1}^{\Phi_{0}, \varphi_{1}} f & =\int_{\mathbb{R}^{2 d}} 1\left\langle f, \pi(z) \Phi_{0}\right\rangle \varphi_{1} d z \\
& =\left\langle\varphi_{1}, \Phi_{0}\right\rangle f=\left|\left\langle\varphi_{1}, \varphi_{2}\right\rangle\right|^{2} f=f .
\end{aligned}
$$

The remainder $E_{1}$ is compact on $M_{\mu}^{p, q}$ because of assumption (ii) on $a$ and assumption (iii) on the windows and Theorem 5.1(ii). This means that $A_{1 / a}^{\varphi_{2}, \varphi_{1}}$ is a left parametrix for $A_{a}^{\varphi_{1}, \varphi_{2}}$.

Construction of a right parametrix of $A_{a}^{\varphi_{1}, \varphi_{2}}$. The construction of a right parametrix is similar. Since we apply Proposition 6.2 to $M_{\mu m}^{p, q}$ instead of $M_{\mu}^{p, q}$, we have to replace the pair of weights $(w, \mu)$ in the hypotheses by the pair $(v w, \mu m)$. Then, by the symmetric formula (44), we obtain the composition formula on $M_{\mu m}^{p, q}$ :

$$
A_{a}^{\varphi_{1}, \varphi_{2}} A_{1 / a}^{\varphi_{2}, \varphi_{1}}=A_{1}^{\Phi_{0}, \varphi_{2}}+\widetilde{E}_{1}=I+\widetilde{E}_{1}
$$

Again by Theorem 5.1(ii) $\widetilde{E}_{1}$ is a compact operator on $M_{\mu m}^{p, q}$, and thus $A_{1 / a}^{\varphi_{2}, \varphi_{1}}$ is the right parametrix for $A_{a}^{\varphi_{1}, \varphi_{2}}$.

Altogether we have shown that $A_{a}^{\varphi_{1}, \varphi_{2}}$ is a Fredholm operators between $M_{\mu}^{p, q}$ and $M_{\mu m}^{p, q}$. Likewise, $A_{1 / a}^{\varphi_{2}, \varphi_{1}}$ is Fredholm between $M_{\mu m}^{p, q}$ and $M_{\mu}^{p, q}$.

Theorem 7.1 applies to the standard weight functions, such as $\left(1+|z|^{2}\right)^{s / 2}$ or $(1+|z|)^{s}$, and also to submultiplicative weights satisfying a condition of the form 
$\left|\partial_{j} v(z)\right| \leq v(z)^{\tau}$ for some $\tau, 0<\tau<1$. However, as written, our main theorem does not seem to work for the subexponential weights $v(z)=e^{a|z|^{b}}, 0<b<1$, because

$$
\left(\partial_{j} e^{a|z|^{b}}\right) e^{-a|z|^{b}}=-a|z|^{b-1} \frac{z_{j}}{|z|}
$$

possesses a mild singularity at 0 and is not bounded in a neighbourhood of 0 . The next result shows that this difficulty can be circumvented easily, because the decisive property of $a$ in Theorem 6.1 is the asymptotic behavior at $\infty$ and not the local behavior at 0 .

Corollary 7.2. The conclusions of Theorem 7.1 hold if condition (ii) on the symbol a is replaced by the weaker condition:

$\left(i i^{\prime}\right)$ there exists a compact set $K \subset \mathbb{R}^{2 d}$ such that,

$$
\left(1-\chi_{K}\right)\left(\partial_{j} a\right) m \in L^{0}\left(\mathbb{R}^{2 d}\right), \quad j=1, \ldots, 2 d .
$$

Proof. We first find the left-inverse of $A_{a}^{\varphi_{1}, \varphi_{2}}$. Let $\psi$ be a test function in $\mathcal{D}$ such that $\psi(z)=1$ for $z \in K$. Then the product $A_{1 / a}^{\varphi_{2}, \varphi_{1}} A_{a}^{\varphi_{1}, \varphi_{2}}$ can be recast as

$$
A_{1 / a}^{\varphi_{2}, \varphi_{1}} A_{a}^{\varphi_{1}, \varphi_{2}}=A_{1 / a}^{\varphi_{2}, \varphi_{1}} A_{a \psi}^{\varphi_{1}, \varphi_{2}}+A_{(1 / a) \psi}^{\varphi_{2}, \varphi_{1}} A_{a(1-\psi)}^{\varphi_{1}, \varphi_{2}}+A_{(1 / a)(1-\psi)}^{\varphi_{2}, \varphi_{1}} A_{a(1-\psi)}^{\varphi_{1}, \varphi_{2}}
$$

Since both functions $a \psi$ and $(1 / a) \psi$ are bounded with compact support, Lemma 4.1 guarantees that the corresponding operators are compact on $M_{\mu}^{p, q}$. Thus, the first two terms of the right-hand side of (48) are compact operators on $M_{\mu}^{p, q}$. To treat the third term, we apply the expansion formula of Theorem 6.1 with $N=1$ and we obtain

$$
\begin{aligned}
A_{(1 / a)(1-\psi)}^{\varphi_{2}, \varphi_{1}} A_{a(1-\psi)}^{\varphi_{1}, \varphi_{2}} & =A_{(1-\psi)^{2}}^{\Phi_{0}, \varphi_{1}}+E_{1} \\
& =I+\overline{\left\langle\varphi_{1}, \varphi_{2}\right\rangle} A_{-2 \psi+\psi^{2}}^{\varphi_{2}, \varphi_{1}}+E_{1} .
\end{aligned}
$$

Since $\left(i i^{\prime}\right)$ implies that $a(1-\psi)$ satisfies condition (45), the remainder $E_{1}$ is compact on $M_{\mu}^{p, q}$ whereas the compactness of the operator $A_{-2 \psi+\psi^{2}}^{\varphi_{2}, \varphi_{1}}$ on $M_{\mu}^{p, q}$ follows again by Lemma 4.1 .

We have shown that $A_{1 / a}^{\varphi_{2}, \varphi_{1}} A_{a}^{\varphi_{1}, \varphi_{2}}=I+R$, for some compact operator $R$ and thus have obtained a left parametrix for $A_{a}^{\varphi_{1}, \varphi_{2}}$. The argument for the right parametrix is similar.

REMARK: It is natural to conjecture that for symbols of the form $a=1 / \mathrm{m}$ the localization operator $A_{a}^{\varphi_{1}, \varphi_{2}}$ is an isomorphism between $M^{p, q}$ and $M_{m}^{p, q}$. Under very restrictive conditions ( $m$ depends only on one variable, or $m$ is hypoelliptic and grows at most polynomially) this has been proved in $[14,7]$ and others. Using completely different techniques (Banach algebra methods and spectral invariance) the following can be shown: assume that $a$ is measurable and $0<A \leq a(z) \leq$ $B<\infty$ for almost all $z \in \mathbb{R}^{2 d}$ and $\varphi_{1}, \varphi_{2} \in M_{v}^{1}$, then $A_{a}^{\varphi_{1}, \varphi_{2}}$ is an isomorphism on $M_{m}^{p, q}$ for $1 \leq p, q \leq \infty$ and all $m \in \mathcal{M}_{v}$ [19]. In contrast to existing results, no smoothness conditions are required on the symbol $a$ in this case. 


\section{ACKNOWLEDGEMENTS}

The authors would like to thank Prof. Luigi Rodino for fruitful conversations and comments. This work was completed while both authors were visiting the Erwin Schrödinger Institute in Vienna. Its hospitality and great working conditions are gratefully acknowledged.

\section{REFERENCES}

[1] H. Ando and Y. Morimoto. Wick calculus and the Cauthy problem for some dispersive equations. Osaka J. Math., 39(1):123-147, 2002.

[2] A. Benyi, K. Gröchenig, C. Heil, and K. Okoudjou. Modulation spaces and a class of bounded multilinear pseudodifferential operators. J. Operator Theory, 2005. To appear.

[3] F. A. Berezin. Wick and anti-Wick symbols of operators. Mat. Sb. (N.S.), 86(128):578-610, 1971.

[4] G. Björk. Linear partial differential operators and generalized distributions. Ark. Mat., 6:351407, 1966.

[5] P. Boggiatto, E. Buzano, and L. Rodino. Global hypoellipticity and spectral theory, volume 92 of Mathematical Research. Akademie Verlag, Berlin, 1996.

[6] P. Boggiatto, E. Cordero, and K. Gröchenig. Generalized Anti-Wick operators with symbols in distributional Sobolev spaces. Integral Equations and Operator Theory, 48:427-442, 2004.

[7] P. Boggiatto, J. Toft. Embeddings and compactness for generalized Sobolev-Shubin spaces and modulation spaces Appl. Anal., 84(3):269-282, 2005.

[8] A. Córdoba and C. Fefferman. Wave packets and Fourier integral operators. Comm. Partial Differential Equations, 3(11):979-1005, 1978.

[9] E. Cordero and K. Gröchenig. Necessary Conditions for Schatten Class Localization Operators. To appear in Proc. Amer. Math. Soc.

[10] E. Cordero and K. Gröchenig. Time-frequency analysis of Localization operators. J. Funct. Anal., 205(1):107-131, 2003.

[11] E. Cordero and L. Rodino. Wick calculus: a time-frequency approach. Osaka J. Math., 42(1):43-63, 2005.

[12] I. Daubechies. Time-frequency localization operators: a geometric phase space approach. IEEE Trans. Inform. Theory, 34(4):605-612, 1988.

[13] J. Du and M. W. Wong. A product formula for localization operators. Bull. Korean Math. Soc., 37(1):77-84, 2000.

[14] H. G. Feichtinger. Modulation spaces on locally compact abelian groups. In Proceedings of "International Conference on Wavelets and Applications" 2002, pages 99-140, Chennai, India, 2003. Updated version of a technical report, University of Vienna, 1983.

[15] H. G. Feichtinger and K. Gröchenig. Banach spaces related to integrable group representations and their atomic decompositions. I. J. Functional Anal., 86(2):307-340, 1989.

[16] G. B. Folland. Harmonic analysis in phase space. Princeton Univ. Press, Princeton, NJ, 1989.

[17] I. Gel'fand, D. Raikov, and G. Shilov. Commutative normed rings. Chelsea Publishing Co., New York, 1964.

[18] K. Gröchenig. Foundations of Time-Frequency Analysis. Birkhäuser, Boston, 2001.

[19] K. Gröchenig. Almost diagonalization of operators by frames and pseudodifferential operators. Lectures Notes, May 2005.

[20] K. Gröchenig and C. Heil. Modulation spaces and pseudodifferential operators. Integral Equations Operator Theory, 34(4):439-457, 1999.

[21] K. Gröchenig and G. Zimmermann. Hardy's theorem and the short-time Fourier transform of Schwartz functions. J. London Math. Soc., 63:205-214, 2001. 
[22] L. Hörmander. The analysis of linear partial differential operators. I. Springer-Verlag, Berlin, second edition, 1990. Distribution theory and Fourier analysis.

[23] L. Hörmander. The analysis of linear partial differential operators. III, volume 274 of Grundlehren der Mathematischen Wissenschaften [Fundamental Principles of Mathematical Sciences]. Springer-Verlag, Berlin, 1994.

[24] N. Lerner. The Wick calculus of pseudo-differential operators and energy estimates. In New trends in microlocal analysis (Tokyo, 1995), pages 23-37. Springer, Tokyo, 1997.

[25] N. Lerner. The Wick calculus of pseudo-differential operators and some of its applications. Cubo Mat. Educ., 5(1):213-236, 2003.

[26] S. Pilipović and N. Teofanov. Pseudodifferential operators on ultra-modulation spaces. J. Funct. Anal., 208(1):194-228, 2004.

[27] J. Ramanathan and P. Topiwala. Time-frequency localization via the Weyl correspondence. SIAM J. Math. Anal., 24(5):1378-1393, 1993.

[28] M. A. Shubin. Pseudodifferential operators and spectral theory. Springer-Verlag, Berlin, second edition, 2001.

[29] D. Tataru. On the Fefferman-Phong inequality and related problems. Comm. Partial Differential Equations, 27(11-12):2101-2138, 2002.

[30] M. E. Taylor. Pseudodifferential operators. Princeton Univ. Press, Princeton, NJ, 1981.

[31] J. Toft. Continuity properties for modulation spaces, with applications to pseudo-differential calculus. I. J. Funct. Anal., 207(2):399-429, 2004.

[32] J. Toft. Continuity properties for modulation spaces, with applications to pseudo-differential calculus. II. Ann. Global Anal. Geom., 26(1):73-106, 2004.

[33] M. W. Wong. Wavelet transforms and localization operators, volume 136 of Operator Theory: Advances and Applications. Birkhäuser Verlag, Basel, 2002.

Department of Mathematics, University of Torino, Italy

Faculty of Mathematics, University of Vienna, Nordbergstrasse 15, A-1090 ViENNA, Austria

E-mail address: cordero@dm.unito.it,karlheinz.groechenig@univie.ac.at 